\title{
Evolution of group I introns in Porifera: new evidence for intron mobility and implications for DNA barcoding
}

\author{
Astrid Schuster ${ }^{1}$, Jose V. Lopez ${ }^{2}$, Leontine E. Becking ${ }^{3,4}$, Michelle Kelly ${ }^{5}$, Shirley A. Pomponi ${ }^{6}$, Gert Wörheide ${ }^{1,7,8}$, \\ Dirk Erpenbeck ${ }^{1,8^{*}}$ (i) and Paco Cárdenas ${ }^{9^{*}}$
}

\begin{abstract}
Background: Mitochondrial introns intermit coding regions of genes and feature characteristic secondary structures and splicing mechanisms. In metazoans, mitochondrial introns have only been detected in sponges, cnidarians, placozoans and one annelid species. Within demosponges, group I and group II introns are present in six families. Based on different insertion sites within the cox 1 gene and secondary structures, four types of group I and two types of group II introns are known, which can harbor up to three encoding homing endonuclease genes (HEG) of the LAGLIDADG family (group I) and/or reverse transcriptase (group II). However, only little is known about sponge intron mobility, transmission, and origin due to the lack of a comprehensive dataset. We analyzed the largest dataset on sponge mitochondrial group I introns to date: 95 specimens, from 11 different sponge genera which provided novel insights into the evolution of group I introns.
\end{abstract}

Results: For the first time group I introns were detected in four genera of the sponge family Scleritodermidae (Scleritoderma, Microscleroderma, Aciculites, Setidium). We demonstrated that group I introns in sponges aggregate in the most conserved regions of cox 1 . We showed that co-occurrence of two introns in cox 1 is unique among metazoans, but not uncommon in sponges. However, this combination always associates an active intron with a degenerating one. Earlier hypotheses of HGT were confirmed and for the first time VGT and secondary losses of introns conclusively demonstrated.

Conclusion: This study validates the subclass Spirophorina (Tetractinellida) as an intron hotspot in sponges. Our analyses confirm that most sponge group I introns probably originated from fungi. DNA barcoding is discussed and the application of alternative primers suggested.

Keywords: Porifera, Tetractinellida, cox1, HGT, VGT, homing endonuclease gene (HEG), LAGLIDADG, group I intron, DNA barcoding

\section{Background}

Mobile introns are self-splicing DNA sequences that play a major role in genome evolution. Group I and group II introns are distinguished based on their splicing mechanisms and secondary structures. Apart from unique splicing mechanisms, differences between group I and group

\footnotetext{
* Correspondence: erpenbeck@lmu.de; paco.cardenas@fkog.uu.se ${ }^{1}$ Department of Earth- \& Environmental Sciences, Palaeontology and Geobiology, Ludwig-Maximilians-Universität München, Richard-Wagner-Str. 10, 80333 Munich, Germany

${ }^{9}$ Department of Medicinal Chemistry, Division of Pharmacognosy, BioMedical Center, Uppsala University, Husargatan 3, 75123 Uppsala, Sweden

Full list of author information is available at the end of the article
}

II introns were observed within the core regions of their secondary structures. Depending on these structural characteristics, group I introns have been further categorized into IA-IE classes. Group II introns constitute up to six stem-loop domains and are classified I-VI respectively (e.g., [35]). Group I and group II introns often contain open reading frames (ORFs) in their loop regions [70], which can encode for different site-specific homing endonuclease genes (HEGs). The majority of group I introns include HEGs, which have a conservative single or a double motif of the amino-acid sequence LAGLIDADG. In contrast group II introns encode in most cases a 
reverse transcriptase-like (RT) ORF (e.g., [36]). Group I and group II introns are found in all domains of life: group I introns are present in bacterial, organellar, bacteriophage and viral genomes as well as in the nuclear rDNA of eukaryotes. Group II introns have a similar distribution, but are not known from the nuclear rDNA (e.g., [33]). More specifically, group I and/or group II introns are found, e.g., in eukaryotic viruses [92], slime molds [45], choanoflagellates [7], the annelid Nephtys sp. [84], red algae [8], brown algae [25] and plants: green algae [85], liverworts $[58,66,67]$ and different angiosperms [58, 66, 67]. Group II introns seem to thrive especially in plants [60], whereas the largest abundance of group I introns currently occurs within fungi $[23,54,68]$. As an example, the mitochondrial (mt) genome of the fungus Ophioscordyceps sinensis harbors 44 group I introns and six group II introns, accounting for $68.5 \%$ of its $\mathrm{mt}$ genome nucleotides. Here, 12 out of 44 group I introns and only one out of six group II introns are located in the cytochrome c oxidase subunit 1 (cox1) gene [54], an acknowledged insertion hotspot for $\mathrm{mt}$ group I introns [23].

More recently, group I introns have been discovered in the cox 1 of early branching metazoan phyla: Placozoa $[9,17,76]$, Cnidaria $[27,31]$ as well as Porifera [21, 29, $65,82,90]$. Group II introns are rarer, and found in the cox1 of Placozoa [17, 76], and in one demosponge species of the order Axinellida (referred to as Cymbaxinella verrucosa) [43]. In Porifera, group I introns have only been recorded from Demospongiae and Homoscleromorpha and, like in group II introns, always in the cox 1 gene, with only occasional double insertions (Fig. 1). The current nomenclature of sponge group I and group II introns is based on the intron insertion site positions in reference to the Amphimedon queenslandica cox 1 gene (DQ915601) [82]. In Homoscleromorpha, three different intron positions (714, 723 and 870; Fig. 1) are known for three species of the family Plakinidae [29]. Within the demosponge subclass Verongimorpha intron 723 is detected in one species (Aplysinella rhax) of the order Verongiida [21]. Most intron insertions have been found within the demosponge subclass Heteroscleromorpha, in the orders Agelasida, Axinellida [43] and especially Tetractinellida [82]. In the Tetractinellida, group I introns are currently known in five sponge species belonging to three genera (Cinachyrella, Tetilla and Stupenda) and inserted at four mtDNA intron positions: 387, 714, 723 and 870 [47, 65, 82] (Fig. 1). To this date, all sponge group I introns encode a HEG with two LAGLIDADG motifs [21, 29, 47, 82] with the exception of intron 714 in Plakinastrella sp. and intron 870 in Agelas oroides and Axinella polypoides, in which no ORF was detected [29, 43, 52, 90]. Intriguingly, Tetractinellida introns are currently only detected in the families Tetillidae [82] and Stupendidae [47].

Fungi and Placozoa have been proposed as possible donors for group I introns among sponges [43, 47, 65]. However, these findings await corroboration with a broader and more comprehensive taxon set. Intron/HEG phylogenetic analyses and group I/II intron secondary structures are the basis for different scenarios on the

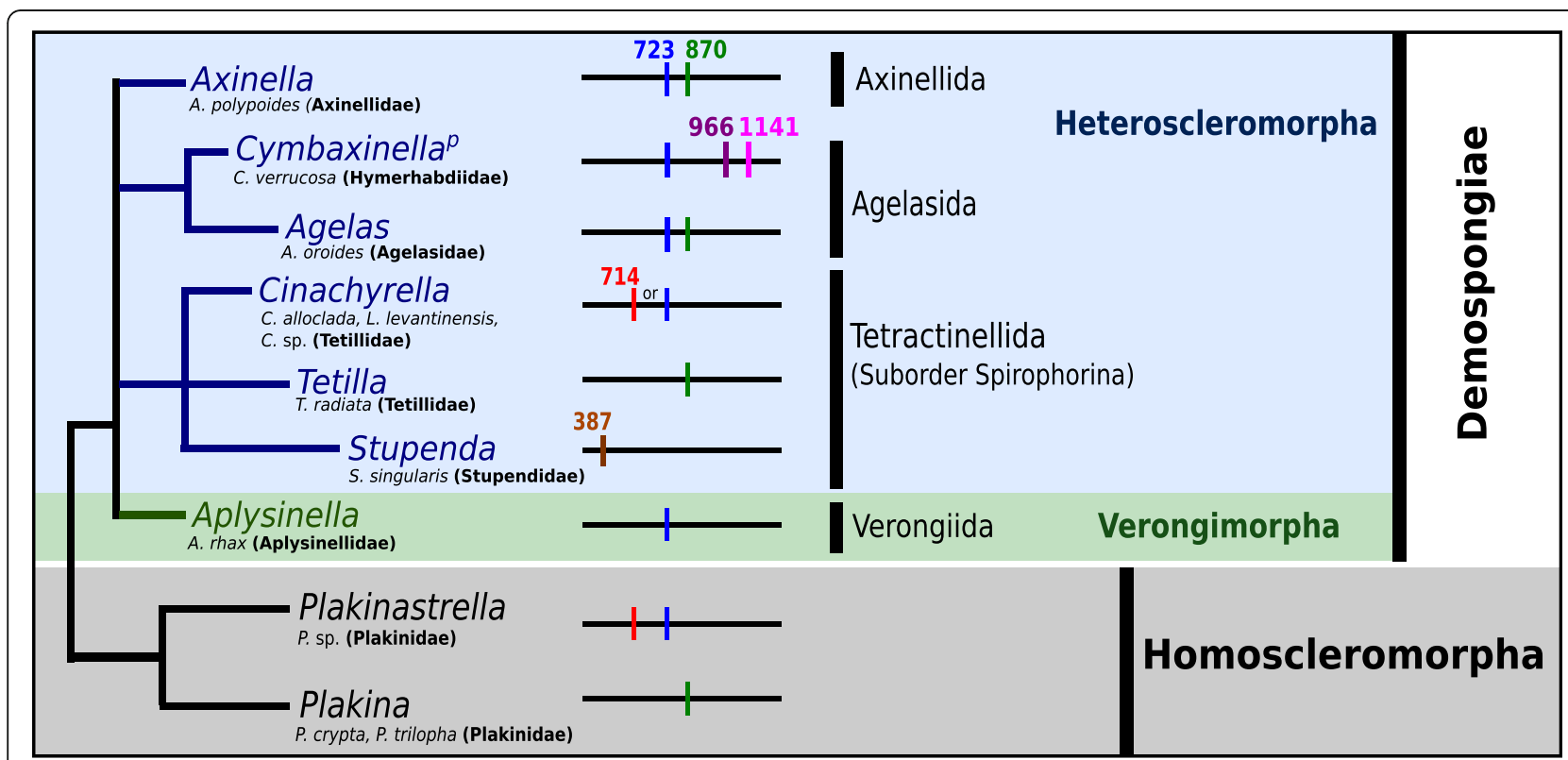

Fig. 1 Simplified sponge phylogeny highlighting currently known group I and II intron insertion sites. Horizontal black lines with colored vertical bars and numbers (blue: intron 723, green: intron 870, red: intron 714, brown: intron 387, purple: intron 966, pink: intron 1141) behind the taxa names represent different intron locations within cox 1 
origin of introns within sponges [43, 47, 82]. The presence of independent horizontal gene transfers (HGT) for introns is supported by their haphazard distribution over phylogenetically distant sponge groups [21, 43, 82]. Vertical gene transfer (VGT) of introns is assumed among closely related taxa, but never confirmed due to the lack of comprehensive taxon sampling [82].

To gain new insights into the evolution of sponge introns we required an intron-rich taxonomic group. Based on earlier studies on sponge introns, the order Tetractinellida represents an obvious target. Other lines of evidence support this choice, such as unsuccessful attempts to amplify cox 1 in this group with standard protocols $[10,72,81]$, potentially due to introns in the relevant primer regions [82]. Consequently, this study focuses on tetractinellid cox1 mitochondrial data to broaden our knowledge on mt intron evolution in this early-branching metazoan phylum.

The data from this "intron-hotspot taxon" presented here constitutes the most representative dataset to target specific questions pivotal to understand intron structure and distribution including activity and mobility. Importance of HGT or VGT or a combination of both will be addressed. Additionally, current hypotheses on the origin of sponge mitochondrial introns will be discussed by comparing intron data across other phyla.

\section{Results}

Mitochondrial intron diversity and characteristics in tetractinellid sponges

The current study comprises the largest dataset of sponge mitochondrial introns to date (95 sequences of which 72 are new), encompassing 13 different sponge genera. All 72 newly sequenced introns were group I introns of the class IB, and all encoded a HEG of the LAGLIDADG family, except for intron 723 of Aciculites sp.1, where no HEG was observed. A double motif of the LAGLIDADG domain was located in all introns, if the sequence was not degenerated or without a HEG. Different intron lengths were observed for different species, and an overview of the different initiation and stop codons of all HE ORFs is given in Fig. 2. All introns possessed start and stop codons in the same frame as the $5^{\prime}$ exon, except intron 714 in Plakinastrella sp. Additionally, uninterrupted ORFs in the same $5^{\prime}$ exonic reading frame were observed for all sequences unless introns were degenerated or without HEG. Initiation and stop codons varied among the intron HEGs. For example the HEG of intron 387 potentially starts with a GTG initiation codon at position 19 of the intron, and not TTA (position 16) as suggested previously [47]. The HEG of intron 714 potentially starts with TTG as its initial codon (position 27). Instead of TTA (position 1) as suggested by Rot et al. [65], all intron 723 HEGs potentially start with ATT (position 10). In the intron HEG 870 we have ATT (position 24 for all) and GTG (position 9 for Plakina and 21 for Tetilla) as initiation codons. The stop codon for most HE ORFs was TAG or TAA except for Cinachyrella sp. 2 and Setidium sp.1 (intron 723), where truncated HEGs were found.

We discovered more introns in the Spirophorina at positions 714, 723 and 870 (Fig. 2); no intron at position 387 was found. As an example, intron 714 sequences were generated for five more Cinachyrella sp. 2 taxa; four from the Indian Ocean (Kenya, Myanmar) and one from the southwest-Pacific (Indonesia). Cinachyrella species are previously known to have only one intron insertion at a time (either 714 or 723 ). However, our study reveals that both introns 714 and 723 can occur together in coxl, e.g., in Cinachyrella sp. 2 from marine lakes (RMNH POR11161) and mangroves (RMNH POR11187). Intron 723 was sequenced from 11 different Cinachyrella species, and it is particularly present in the Cinachyrella alloclada complex. In total this study contains 42 sequences of Cinachyrella alloclada (intron 723) from the western Atlantic, the Caribbean Sea and the Gulf of Mexico. We added six additional intron 723 sequences including C. cf. anomala, C. cf. providentiae, C. porosa (all Indonesia), C. sp. 3 (Red Sea), C. sp. 4 (Morocco) and C. sp. 5 (Taiwan). The resulting Cinachyrella dataset covers subtropical-tropical areas from 1 to $90 \mathrm{~m}$ depth.

For the first time we discovered intron 723 in the Scleritodermidae (Microscleroderma, Aciculites, Setidium and Scleritoderma). Intron 870 was found in Tetilla quirimure from Brazil and Microscleroderma herdmani from the Indian Ocean (Mauritius), and the Pacific (Philippines and Hawaii). Huchon et al. [43] located intron 723 in combination with intron 870 in two families (Axinellidae and Agelasidae), while our study reveals this combination in three scleritodermid genera (Microscleroderma, Aciculites, Setidium).

\section{Comparative intron and exon phylogenies of Tetractinellida}

Phylogenetic reconstructions of the $\operatorname{cox} 1$ exon and the intron revealed a patchy distribution of intron insertions among the Scleritodermidae and Tetillidae and different levels of congruence among intron and exon phylogenies.

\section{Family Tetillidae}

The relationships of major clades (Fig. 3) were in concordance with a previous study [81]. Unlike Cinachyrella, Tetilla appeared monophyletic in reconstructions with five species (T. radiata, T. japonica, $T$. quirimure, $T$. dactyloidea and $T$. muricyi). Tetilla radiata (intron 870 ) is sister to the intron-lacking Tetilla japonica (posterior probability $[\mathrm{PP}]=1.00 /$ bootstrap support $[\mathrm{BS}]=98$ ). As shown by Szitenberg et al. [81], 


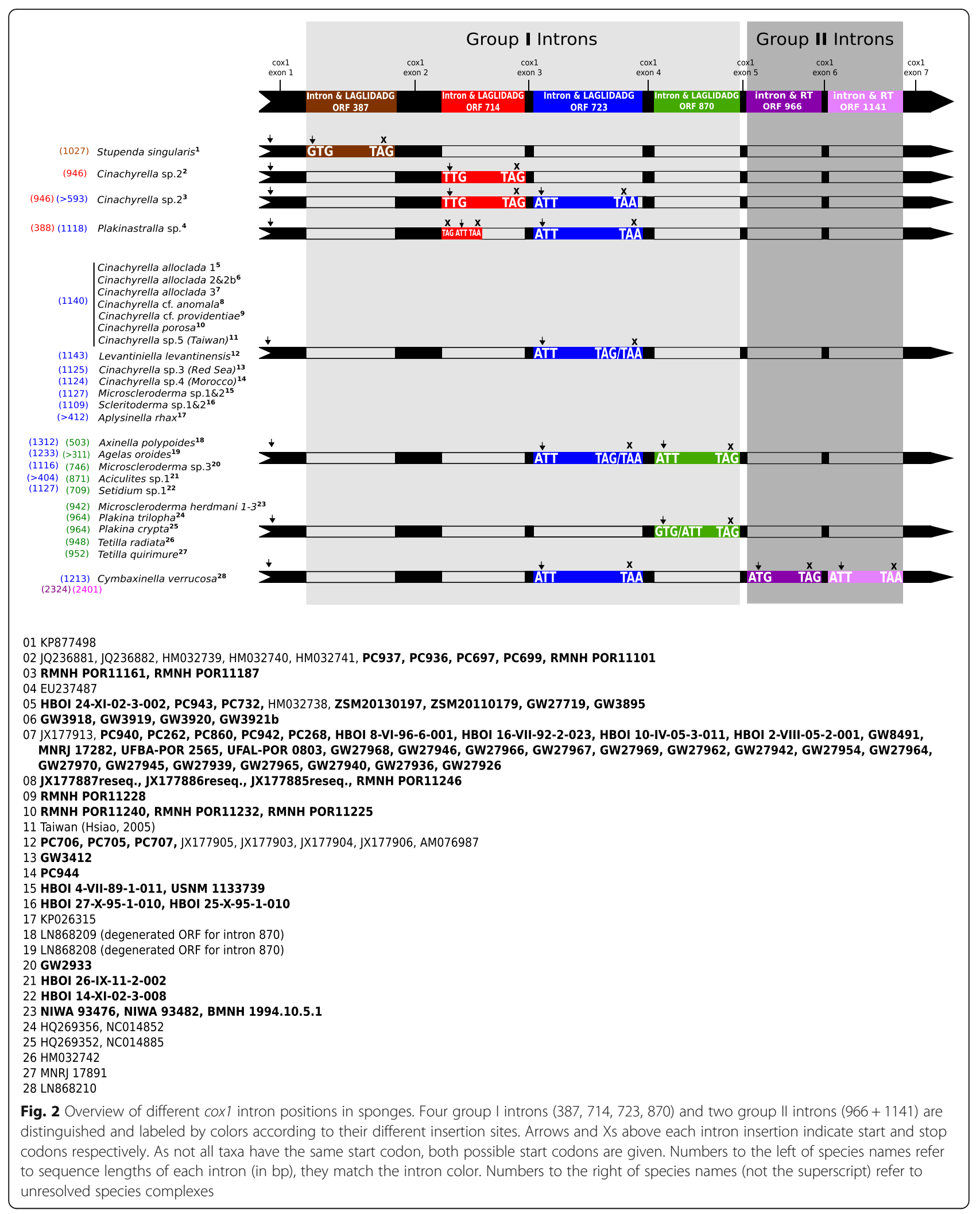


Bayesian Posterior $\mid$ Maximum Likelihood

$$
\begin{array}{c|c}
\text { Probabilities } & \text { Bootstrap } \\
0.95-1.00 \square & \square 5-100 \\
0.75-0.94 \square & 60-74 \\
<0.75 \square & \square<60
\end{array}
$$

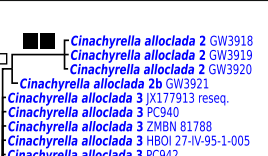

-Cinachyrella alloclada 3 ZMBN 81788 Cinachyrella alloclada 3 PC942
Cinachyrella alloclada 3 PC26

Cinachyrella alloclada 3 HBOO 8.V-1.96-6-001

Cinachyrella alloclada 3 GW84991
Cinachyrella alloclada 3 GW27968

-Cinachyrella alloclada 3 GW27971

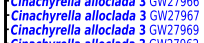

- Cinachyrerllla allocladlada $3 \mathrm{GW} 27$.

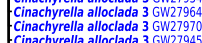

a

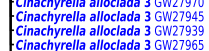

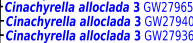

Cinachyrella alloclada 3 GW27926 $\quad$ Cinachyrella cf. anomala 3 | X1778855 reseq.

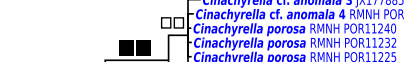

Cinachyrella anomala J X17788

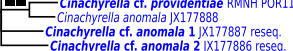

- Cinachyrella sp. 3 GW3412

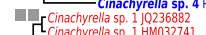

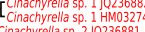

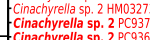

Cinachyrella sp. 2 BELUM:Mc8286

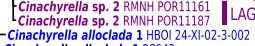

a.

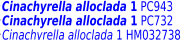

Cinachyrella alloclada 1 ZSM2013019
cinachyrella alloclada 1 ZSM20110179
cinachyrella alloclada 1 GW W77719
Cinachyrella alloclada 1 GW3895

$\infty \stackrel{1}{n}$

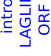

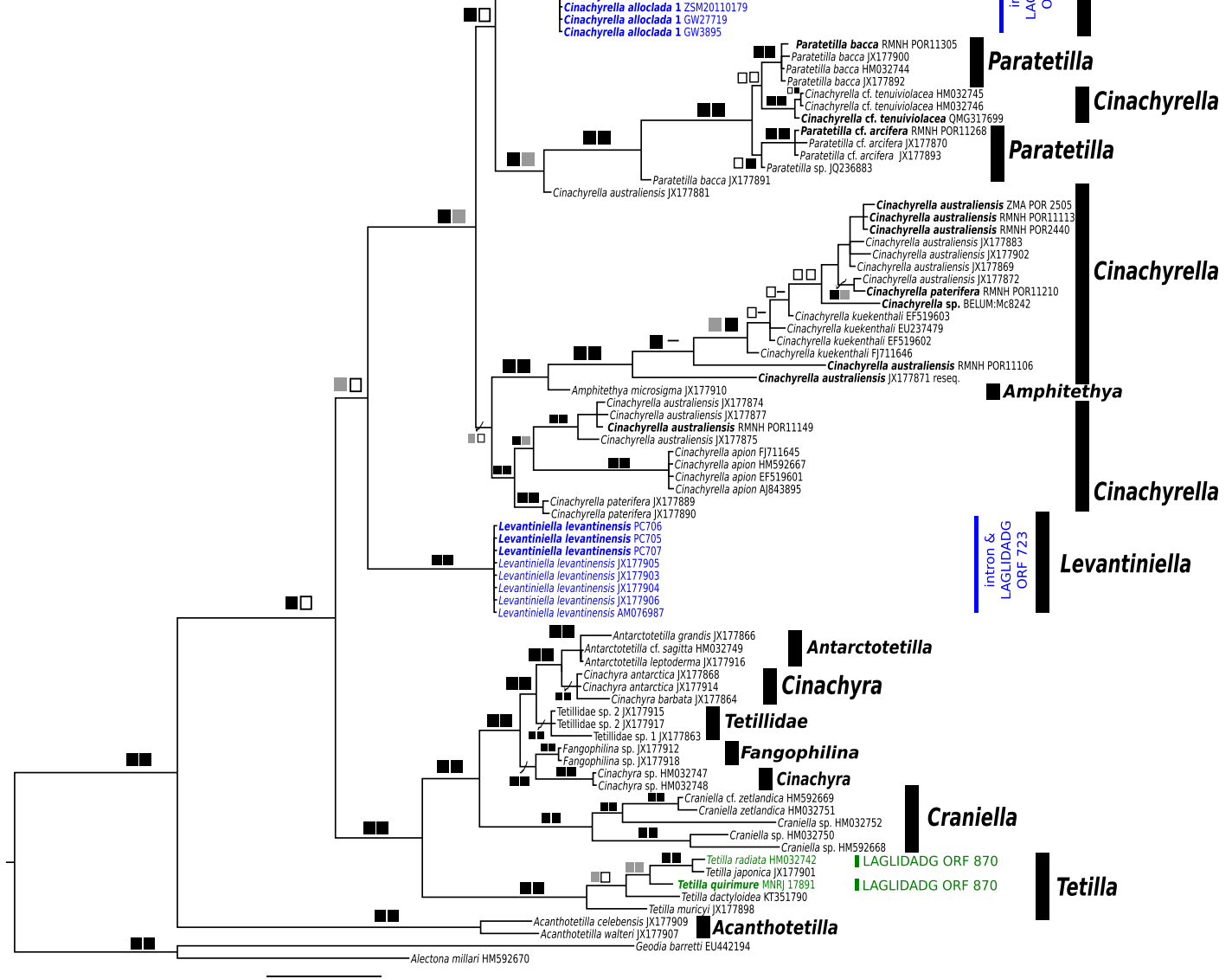

0.02

Fig. 3 (See legend on next page.) 
(See figure on previous page.)

Fig. 3 Coxl (introns excluded) bayesian inference (MrBayes, GTR $+G+$ I model) phylogeny of the family Tetillidae. The maximum likelihood (RAxML) tree is congruent. Squares represent node supports. Black squares: $\mathrm{PP}=0.95-1.00, \mathrm{BP}=75-100$. Dark gray squares: $\mathrm{PP}=0.75-0.94, \mathrm{BP}=$ 60-74. White squares: PP $<0.75, \mathrm{BP}<60$. Numbers behind each taxa are GenBank accession numbers or voucher numbers (for new sequences). Sequences generated in this study are in bold. Color code follows Fig. 2 and corresponds to the different intron insertion positions, except two Cinachyrella sp. 2 species, which are marked in purple as they possess two introns $(714+723)$

our analysis supported the early-branching position of the intron 723-bearing Levantiniella levantinensis with respect to the Cinachyrella/Paratetilla/Amphitethya clade. Intron 723-bearing Tetillidae did not form a monophyletic group due to the position of L. levantinensis and the presence of a highly supported (PP 1.00/BS 100) clade of intron 714 bearing species consisting of $C$. sp. 1 (intron 714 only) and $C$. sp. 2 (with intron 714 only or in combination with intron 723). There were no genetic differences in cox1 between Cinachyrella sp. 2 bearing one (714) or two (714 and 723) introns.

\section{Family Scleritodermidae}

The cox 1 exon phylogeny (Fig. 4) corroborated the sister group relationship Scleritodermidae/Stupdendidae, previously suggested with $18 \mathrm{~S}$ rDNA data [47]. Results also supported the monophyly of Scleritodermidae and its genera Microscleroderma, Aciculites and Scleritoderma as previously suggested by $18 \mathrm{~S}$ and $28 \mathrm{~S}$ rDNA phylogenies $[61,72]$. Species in these genera displayed different intron distributions (Fig. 4).

\section{Intron + LAGLIDADG Phylogeny}

The intron 723 phylogeny (intron + LAGLIDADG) (Fig. 5) broadly agreed with the corresponding exon phylogeny (Fig. 3), but also displayed several differences crucial for the understanding of sponge intron evolution. Notably, we recovered intron 723 of Cinachyrella sp. 2 in a different clade than for the cox 1 of all Cinachyrella sp. 2, whether they have intron 714 or both $714+723$ (Fig. 3). The clade of introns 723 of Cinachyrella sp. 3 and sp. 4, from the Red Sea and Morocco respectively, were in a sister-group relationship with L. levantinensis whereas in the exon phylogeny these two species branched within the Cinachyrella clade (Fig. 3). These incongruences between the exon and the intron phylogenies were shown to be significant $(p<0.01$, Shimodaira-Hasegawa (SH)-test). One single C. alloclada 1 sequence falls within the C. alloclada 3 clade. This position is regarded as artifactual due to an incomplete intron sequence as retrieved from degraded DNA.

Interestingly, Agelas oroides and Cymbaxinella verrucosa intron sequences grouped within Spirophorina in a highly supported sister group to Cinachyrella introns. The intron + LAGLIDADG phylogenies for 870 and 714 were congruent with the exon phylogeny for all supported clades (Additional file 1).

\section{Secondary structure analyses of introns 723 and 870}

The secondary structures of intron 723 and intron 870 presented the typical RNA fold of a group I intron structure [88], consisting of a P1-P2-P10 substrate domain, a P4-P5P6 scaffold domain and a P3-P7-P8 catalytic domain (Fig. 6). The conserved regions $\mathrm{Q}, \mathrm{P}, \mathrm{S}$, and R, building the core, were found in all of the structures.

Our current study expands our knowledge on Cinachyrella intron 723 [82] thanks to five additional structures predicted for Cinachyrella porosa (RMNH POR11225), Cinachyrella sp. 4 (PC944), Cinachyrella alloclada 2 (GW3920), Cinachyrella cf. providentiae (RMNH POR11228), and Cinachyrella cf. anomala (JX177887) (Additional file 2). The structures of introns 714 and 723 only have a single-stranded P2 region, whereas intron 870 has a double stranded P2 region (Fig. 6, [82]). The LAGLIDADG ORF is always located in the loop of the P8 helix (Fig. 6, Additional file 2).

Intron 723 structural differences between the species are in the P6 and P9 regions. In particular, Cinachyrella sp. 4 from Morocco has reduced helices P9.1c and P9.1d compared to all others. Cinachyrella alloclada 2 differs slightly in the P6, P6a, P6b and P6d regions to other Cinachyrella species. We generated for the first time secondary structures of scleritodermid intron 723 in Microscleroderma sp. 2 (USNM 1133739), Setidium sp. 1 (HBOI 14-XI-02-3-008) and Scleritoderma sp. 2 (HBOI 25-X-95-1-010) (Fig. 6). All three species show a high variability in loops and helices within the P9 region. Only a few differences were observed in the P6 region between the species. The main difference between Cinachyrella and Scleritodermidae intron 723 is the absence of the P6d region in the latter (Fig. 6a, b and c).

The secondary structure of intron 870 was reconstructed for Tetilla quirimure (MNRJ 17891) and Microscleroderma herdmani 3 (BMNH 1994.10.5.1) (Fig. 6d and e). Both taxa contain the known core helices and conserved structures of Q, P, S, and R. Tetilla quirimure intron structure is very similar regarding P6 and P9 regions to the one from Tetilla radiata [82]. The intron of Microscleroderma herdmani, in turn, has a reduced P6a helix and a different P5a region compared to that found in Tetilla.

\section{The LAGLIDADG protein phylogeny}

The sponge LAGLIDADG sequences displayed phylogenetic affinities to four different clades (Fig. 7). LAGLIDADG 


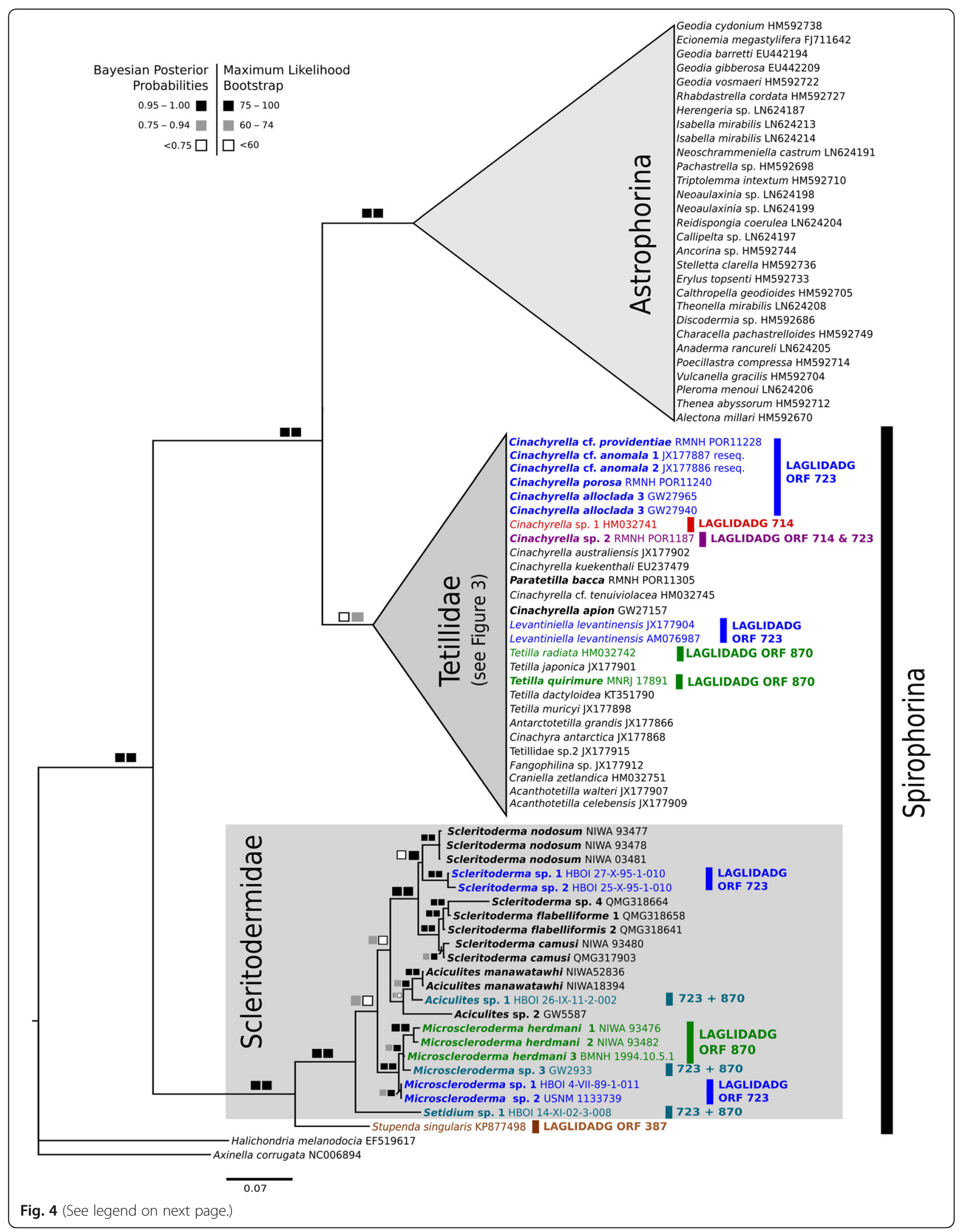


(See figure on previous page.)

Fig. 4 Cox1 (introns excluded) bayesian inference (MrBayes, $G T R+G+I$ model) phylogeny of the Tetractinellida with focus on the family Scleritodermidae. The maximum likelihood (RAxML) tree is congruent. Squares represent node supports. Black squares: $P P=0.95-1.00, B P=75-$ 100. Dark gray squares: $P P=0.75-0.94, B P=60-74$. White squares: $P P<0.75, B P<60$. Numbers after each taxa are GenBank accession numbers or voucher numbers (for new sequences). Sequences generated in this study are in bold (32 sequences). Color code follows Fig. 2, except for three Scleritodermidae taxa, which possess two intron insertions $(723+870)$ and marked in dark-cyan respectively

(intron 387) of Stupenda singularis forms a highly supported sister group relationship with a Fungi/Marchantiophyta clade. Cnidarian LAGLIDADG encoding sequences are only present in Hexacorallia and never revealed in the other subclasses Octocorallia and Ceriantharia. Within Hexacorallia, the sequences of intron 888 are monophyletic and include the orders Actiniaria (sea anemones), Scleractinia (stony corals), Corallimorpharia (corallimorphs) and Antipatharia (black corals) [31]. Notably, there are no sponge LAGLIDADG (intron 888) sequences. In comparison, several scleractinian sequences form a clade with the sponge LAGLIDADG intron 723 sequences.

LAGLIDADG (intron 870) of Plakina/Tetilla and the Scleritodermidae are closely related to Hexacorallia (Zoantharia) LAGLIDADG sequences, but with considerable genetic distance. The genealogical affinities of intron 714 appear unresolved.

\section{Discussion}

\section{Characterisation and mobility mechanisms of group I intron}

In sponges, group I and II introns occur coincidentally and exclusively in the $\operatorname{cox} 1$ gene in a few sponge groups (Fig. 1). The current study specifically targeting sponge mitochondrial cox 1 introns represents the largest sponge $\mathrm{mt}$ intron dataset (95 specimens), encompassing 11 different sponge genera. In accordance with previous studies $[21,43,47,65,82]$, double or single intron insertion sites within the cox 1 gene were observed among different taxa (Fig. 2). Sponge group I intron sequences in most cases contain a putative homing endonuclease (HE) ORF, which encode for a LAGLIDADG-type protein, unless they are degenerated (Fig. 2). Intron mobility is facilitated by those site specific HEs, which conduct double-strand breaks (DSBs) in alleles that lack introns, hence activating intron mobility via a DSB-repair process [2]. Those HEs are known to promote their mobility towards conserved regions [35]. However, only a few studies have investigated the conservation of those introns in their host genes. Swithers et al. [80] analysed the conservation of group I and group II introns in the host genes of vascular plants, protists, fungi, green algae, liverworts and amoeba, but not in animals. In the former, group I introns are preferentially located within conserved regions, whereby group II introns were not shown to remain particularly in conserved sites [80]. In comparison, our conservation analysis (Fig. 8) corroborated these findings by demonstrating that even in early branching metazoans like sponges, group I introns are located in the most conserved regions of their host proteins. At present, sponge group II intron insertions are only known from a single demosponge (Cymbaxinella verru$\cos a$ ) [43], with two group II introns in the 3' region of cox 1 . Our conservation analysis showed that these two group II introns were located in conserved regions (Fig. 8), however, additional data are needed for a better understanding of group II intron conservation and mobility in sponges.

The $\operatorname{cox} 1$ gene in demosponges has the lowest substitution rate of all $\mathrm{mt}$ protein coding genes [90]. In fact, the mitochondrial genomes of the sponge classes Homoscleromorpha and Demospongiae possess features shared with non-metazoan opisthokonts rather than Bilateria such as the presence of intergenic regions, genes of foreign origin, a low substitution rate, selfish elements and introns [50]. Mt introns are also found in plants [28], fungi [44], Placozoa [76], and Hexacorallia $[13,37,74]$ all known to have slow rates of evolution. It is assumed that this lower substitution rate slows down the elimination of ribozyme activities within group I introns, therefore HEGs would degenerate slower in most fungi [23], anthozoans [27, 31] and placozoans [9]. Intron mobility is particularly dependant on secondary structure and therefore mutation pressure, so sponge introns survive in the most conserved mt gene ( $\operatorname{cox} 1)$, and the most conserved regions of this gene (Fig. 8), where their HEG is most likely to degenerate slowly. On the other hand, hexactinellid and particularly calcareous sponges possess an accelerated substitution rate $[51,52]$ and no known mt introns to this date. This correlation between higher mutation rate and absence of mt intron is shared by Cubozoa [77], Ceriantharia [79]; Ctenophora [48, 59], Hydrozoa and Scyphozoa [42]. One exception to this pattern is the apparent lack of introns in Octocorallia, despite their lower substitution rates compared to the intron-bearing Hexacorallia [37, 74]. This might be due to the presence of a unique MutS gene, which encodes a DNA mismatch repair machinery [4], which prevents intron insertions. The mt DNA mismatch repair machinery in sponges remains unknown.

Sponge group I introns consist of complex catalytic ribozymes (RNAs) that fold into a conserved threedimensional core structure of ten helices. Within this structure, the sponge HEGs are found to be always 
Bayesian Posterior | Maximum Likelihood Probabilities Bootstrap

$0.95-1.00 \square \square 75-100$

$0.75-0.94 \square 60-74$

$<0.75 \square \mid \square<60$
Cinachyrella alloclada 2 GW3920

Cinachyrella alloclada 2 GW3918

Cinachyrella alloclada 2 GW3919

Cinachyrella alloclada 2b GW3921

Cinachyrella alloclada 1 HBOI 24-XI-02-3-002

Cinachyrella alloclada 3 HBOI 8-VI-96-6-001

Cinachyrella alloclada 3 UFBA-POR 2565

Cinachyrella alloclada 3 UFAL-POR 0803

Cinachyrella alloclada 3 JX177913 (resequ.)

Cinachyrella alloclada 3 GW8491

Cinachyrella alloclada 3 ZMBN 81788

Cinachyrella alloclada 3 HBOI 27-IV-95-1-005

Cinachyrella alloclada 3 MNRJ17282

Cinachyrella alloclada 3 HBOI 16-VII-92-2-023

Cinachyrella alloclada 3 PC942

Cinachyrella alloclada 3 HBOI 2-VIII-05-2-001

Cinachyrella alloclada 3 PC268

Cinachyrella alloclada 3 PC940

$\square \square$ Cinachyrella alloclada 3 HBOI 10-IV-05-3-011

Cinachyrella alloclada 3 GW27968

Cinachyrella alloclada 3 GW27971

Cinachyrella alloclada 3 GW27966

Cinachyrella alloclada 3 GW27967

Cinachyrella alloclada 3 GW27969

Cinachyrella alloclada 3 GW27962

Cinachyrella alloclada 3 GW27942

Cinachyrella alloclada 3 GW27954

Cinachyrella alloclada 3 GW27964

Cinachyrella alloclada 3 GW27970

Cinachyrella alloclada 3 GW27945

Cinachyrella alloclada 3 GW27939

Cinachyrella alloclada 3 GW27965

Cinachyrella alloclada 3 GW27940

Cinachyrella alloclada 3 GW27936

Cinachyrella alloclada 3 GW27926

- Cinachyrella porosa RMNH POR11232

Cinachyrella cf. anomala 4 RMNH POR11246

a Cinachyrella sp. 2 RMNH POR 11187

Cinachyrella sp. 2 RMNH POR11161

a Cinachyrella sp. 5 Taiwan

Cinachyrella porosa RMNH POR 11240

Cinachyrella porosa RMNH POR 11225

- Cinachyrella cf. anomala 3 JX177885

Cinachyrella cf. anomala 2 JX177886

-Cinachyrella cf. anomala 1 JX177887

Cinachyrella cf. providentiae RMNH POR11228

Cinachyrella alloclada 1 ZSM20110179

Cinachyrella alloclada 1 ZSM20130197

- Cinachyrella alloclada 1 HM032738

Cinachyrella alloclada 1 PC732

Cinachyrella alloclada 1 GW3895

Cinachyrella alloclada 1 GW27719

Cinachyrella alloclada 1 PC943

Levantiniella levantinensis PC706

Levantiniella levantinensis PC705

Levantiniella levantinensis $\mathrm{PC} 707$

$\square$

Levantiniella levantinensis JX177905

Levantiniella levantinensis JX177903

Levantiniella levantinensis JX177904

Levantiniella levantinensis JX177906

Levantiniella levantinensis AM076987

- Cinachyrella sp. 4 PC944

$\left[\begin{array}{l}\text { Cinachyrella sp. } 4 \text { PC944 } \\ \text { Cinachyrella sp. } 3 \text { GW } 3412\end{array}\right.$

Cymbaxinella verrucosa LN868210

-1. Microscleroderma sp. 1 HBOI 4-VII-89-1-011

Microscleroderma sp. 2 USNM 1133739

Microscleroderma sp. 3 GW2933

- Setidium sp. 1 HBOI 14-XI-02-3-008

a Scleritoderma sp. 2 HBOI 25-X-95-1-010

Scleritoderma sp. 1 HBOI 27-X-95-1-010
N-Atlantic (Florida)

Caribbean Sea (Panama)

N-Atlantic (Florida)

N-Atlantic (Florida)

N-Atlantic (Bahamas)

Caribbean Sea (Puerto Rico)

S-Atlantic (Salvador, Brazil)

S-Atlantic (Mar. Deodoro, Brazil)

N-Atlantic (Bahamas)

N-Atlantic (Florida)

Caribbean Sea (Panama)

N-Atlantic (Bahamas)

S-Atlantic (Maceió, Brazil)

Gulf of Mexico (Dry Tortugas)

N-Atlantic (Florida)

N-Atlantic (Florida)

Caribbean Sea (Panama)

N-Atlantic (Florida)

N-Atlantic (Bahamas)

N-Atlantic (Florida)

N-Atlantic (Florida)

N-Atlantic (Florida)

N-Atlantic (Florida)

N-Atlantic (Florida)

N-Atlantic (Florida)

N-Atlantic (Florida)

N-Atlantic (Florida)

N-Atlantic (Florida)

N-Atlantic (Florida)

N-Atlantic (Florida)

N-Atlantic (Florida)

N-Atlantic (Florida)

N-Atlantic (Florida)

N-Atlantic (Florida)

N-Atlantic (Florida)

Pacific (Berau, Indonesia)

Pacific (West Papua, Indonesia)

Pacific (West Papua, Indonesia)

Pacific (Berau, Indonesia)

South China Sea (Taiwan)

Pacific (Berau, Indonesia)

Pacific (Berau, Indonesia)

Pacific (West Papua, Indonesia)

Pacific (West Papua, Indonesia)

Pacific (West Papua, Indonesia)

Pacific (Berau, Indonesia)

Caribbean Sea (Jamaica)

Caribbean Sea (Jamaica)

N-Atlantic (Bahamas)

N-Atlantic (Florida, Keys)

N-Atlantic (Florida, Keys)

Caribbean Sea (Panama)

N-Atlantic (Florida)

Mediterranean Sea (Israel)

Mediterranean Sea (Israel)

Mediterranean Sea (Israel)

Mediterranean Sea (Lebanon)

Mediterranean Sea (Israel)

Mediterranean Sea (Israel)

Mediterranean Sea (Israel)

Mediterranean Sea

N-Atlantic (Morocco)

Red Sea (Saudi-Arabia)

Agelas oroides LN868208

Aplysinella rhax KP026315

Axinella polypoides LN868209 
(See figure on previous page.)

Fig. 5 Intron 723 bayesian inference (MrBayes, GTR $+G+$ I model) phylogeny in sponges. The maximum likelihood (RAxML) tree is congruent. Squares represent node supports. Black squares: $\mathrm{PP}=0.95-1.00, \mathrm{BP}=75-100$. Dark gray squares: $\mathrm{PP}=0.75-0.94, \mathrm{BP}=60-74$. White squares: $\mathrm{PP}<$ $0.75, \mathrm{BP}<60$. Numbers after each taxa are GenBank accession numbers or voucher numbers (cf. Additional file 1). Sequences generated in this study are in bold (63 sequences). Sampling localities for the subtropical-tropical Cinachyrella taxa are given

located within the loop region of the catalytic domain (helix P8, Fig. 6) as in Hexacorallia [31]. HEGs and their intron partners are thought to move either independently from each other [73] or as a single unit [34]. Whether those HEGs are actively expressed or not often depends on their functionality. The functional expression of HEG group I introns and the resulting gains and/or losses are considered as a cyclical process of different stages [30]. Emblem et al. [20] applied this into an evolutionary model for a group I intron in sea anemones and reported five stages: 1) Intron with HEG expressed and fused in frame with the upstream host gene exon; 2) Intron with expressed free-standing HEG; 3) Intron with shortened/degenerated HEG; 4) Intron without a conserved HEG and 5) Exon cox1 without intron. Until now, only a few insights into this evolutionary model were given for sponges. Different stages are observed for intron 723 and intron 870 in different sponge species [43]. However, no detailed information has been provided yet on the potential start and stop codons, which are crucial diagnostic features for their categorisation. The potential start and stop codons, observed in all group I introns (Fig. 2), in addition to the predicted secondary structures (Fig. 6, Additional file 2), provide insights into the respective evolutionary stages of all sponge group I introns. In detail, we classified intron 387 of Stupenda singularis in stage 1. Intron 714 of all Cinachyrella sp. 2 appear in stage 1, and in stage 4 for Plakinastrella sp. due to several start and stop codons and no HEG. Intron 723 is found to be in stage 1 among all Cinachyrella species except C. sp. 2 (see below), which is in concordance with the already published data [43]. Intron 723 in Microscleroderma sp. 1 \& 2 and Scleritoderma sp. $1 \& 2$ are also found to be in stage 1 . A study comparing the length of DNA and RNA in combination with RT-PCR on a Cinachyrella intron 723 from Taiwan (probably Cinachyrella sp. 5) suggests that it can self-splice in vivo or in vitro [12]. It confirms that this particular stage 1 intron 723 is active. We observe intron 723 also in stages 3 and 4 in Aplysinella rhax, Microscleroderma sp. 3, Cinachyrella sp. 2, Setidium sp.1 (degenerated HEG) and Aciculites sp. 1 (short sequence and no HEG) respectively, which rebuts the suggested recent infection of intron 723 in sponges [43]. Intron 870 was at stage 1 for Tetilla radiata, Plakina crypta and Plakina trilopha [43] and now shown for Microscleroderma herdmani 1-3, Tetilla quirimure and Setidium sp. 1. Interestingly, both stage 3-4 intron 870 ( $A$. polypoides, A. oroides) previously described [43] cooccur with stage 1 intron 723 . Also, the only stage 4 intron 714 (Plakinastrella sp.) co-occurs with a stage 1 intron 723. Similarly, all of the stage 3-4 intron 723 (Microscleroderma sp. 3, Cinachyrella sp. 2, Aciculites sp. 1, Setidium sp. 1) co-occur with stage 1 introns (either 714 or 870 ). Overall, two stage 1 introns never co-occur, one of the two is always degenerating. We can therefore hypothesize that the presence of two group I introns is unstable or that maybe the degeneration of one somehow enables the insertion of a different intron. More double-intron-bearing cox 1 sequences are needed to study this further. Moreover, we noted that although Scleractinia (Hexacorallia) possesses intron 723 or 888 , no evidence of double-intron $\operatorname{cox} 1$ sequences in this group is given, which applies for Cnidaria in general. Since double-intron cox1 sequences are also absent in Placozoa, sponges (e.g., demosponges and homoscleromorphs) are to date the only metazoans with doubleintron $\operatorname{cox} 1$ sequences.

\section{HGT versus VGT of group I introns}

The sporadic detections and patchy distributions of group I introns not only among sponges, but also among other Metazoa in e.g., scleractinian corals [27, 31], plants [67] and fungi [45] are the main arguments for HGT.

HGT events for group I introns in sponges were first hypothesized by Rot et al. [65] and later corroborated by other studies, based on major differences between cox 1 and intron phylogeny topologies [82] as well as the occurrence of homologous introns in phylogenetically distantly related sponge groups e.g., homoscleromorphs [90] and demosponges (Verongimorpha and Heteroscleromorpha) [21, 43, 47] (Fig. 1). Our results reveal new cases of intron HGT, this time within the Cinachyrella species (Fig. 9). As an example Cinachyrella sp. 3 (GW3412, Red Sea) and Cinachyrella sp. 4 (PC944, Morocco) are sister to Cinachyrella alloclada 2-3/ Cinachyrella spp. from the Pacific whereas their intron 723 are sister to the intron of Levantiniella levantinensis (Fig. 9). One can therefore hypothesize that an intron 723 of L. levantinensis (or one of its ancestors) invaded the ancestor of Cinachyrella sp. 3 and Cinachyrella sp. 4. In addition, two Cinachyrella sp. 2 specimens from the Pacific (RMNH POR11161 and RMNH POR11187) group together with other conspecifics in the cox 1 phylogeny; however, their introns are closely related to Cinachyrella species from the Pacific. Therefore, we 


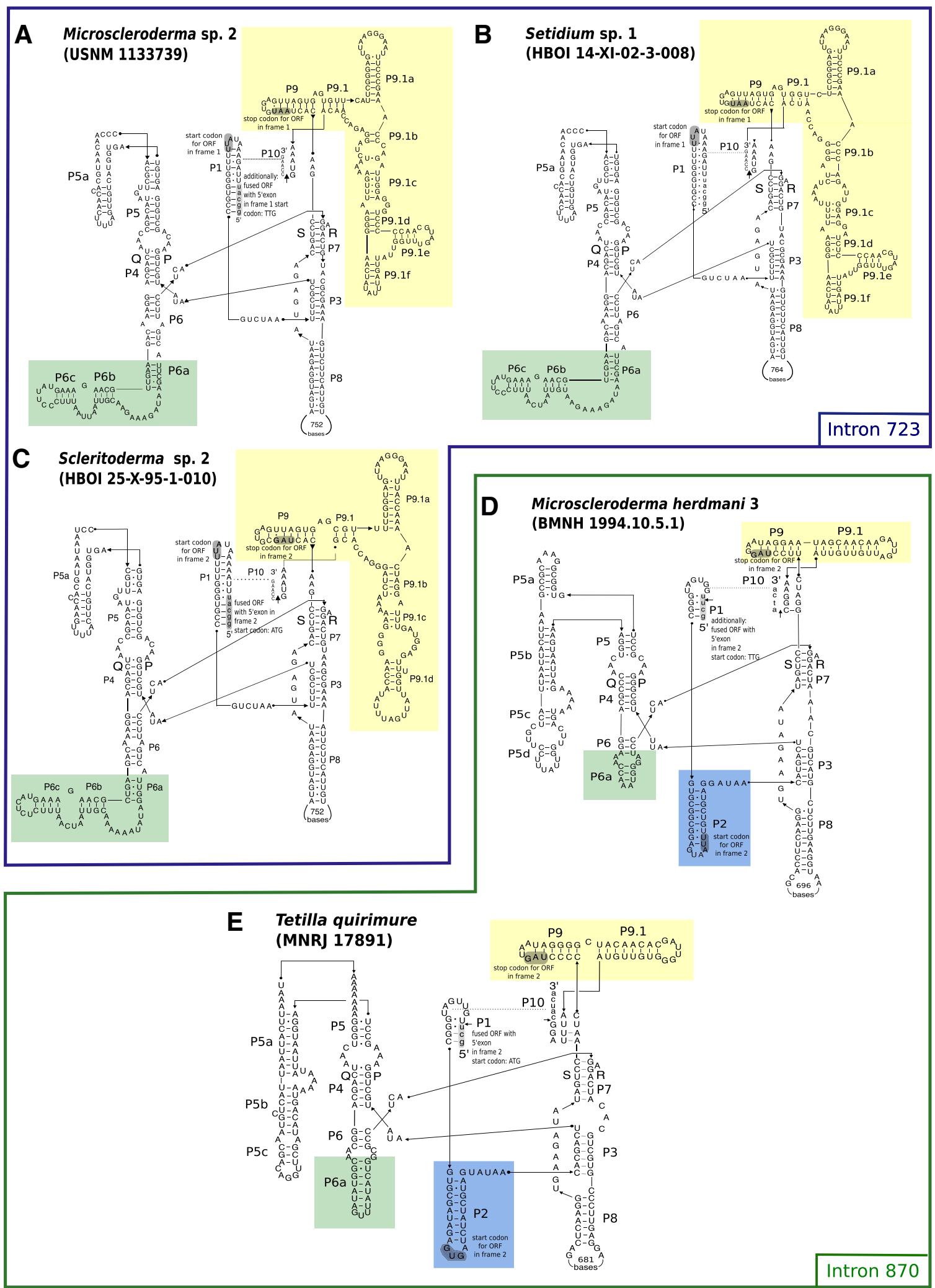

Fig. 6 (See legend on next page.) 
(See figure on previous page.)

Fig. 6 Predicted secondary structures of Microscleroderma sp. 2 (a), Setidium sp. 1 (b), Scleritoderma sp. 2 (c) (group I, IB, intron 723),

Microscleroderma herdmani 3 (d) and Tetilla quirimure (e) (group I, IB, intron 870). Exon bases are in lower-case letters and intron bases in uppercase letters. Paired P1-P10 helices and their conserved sequences $(P, Q, R, S)$ are labeled according to the standard group I intron scheme [88]. The HEGs are present in the loops of their respective P8 helix. For a better comparison the same color scheme as in Szitenberg et al. [82] was used to highlight differences in the P2, P6 and P9 regions. Potential start and stop codons are highlighted in light gray

hypothesize that those two Cinachyrella sp. 2 specimens from marine lakes and mangroves were reinfested by intron 723 after it was primarily lost, which would indicate a "secondary" HGT between C. sp. 2 and the Pacific Cinachyrella spp. (Fig. 9). It also seems that these two specimens are losing their intron 723 again, which is at stage 3 . These hypotheses are significantly corroborated by the SH-test $(p<0.01)$. Another HGT event was found within the family Sceritodermidae: while Setidium sp. 1 is sister to Microscleroderma taxa in the intron phylogeny (Fig. 5), it branches off first of all other Scleritodermidae taxa in the cox 1 phylogeny (Fig. 4). However, the cox 1 topology is poorly supported around Setidium sp., therefore this HGT event is less obvious than in the two previous cases. Although the introns 723 found in the Agelasida (A. oroides and C. verrucosa) are fairly divergent, the intron and the LAGLIDADG phylogenies both suggest that they are phylogenetically related to the Cinachyrella/Levantiniella introns. Indeed, the Agelasida introns share a more recent common ancestor with the Cinachyrella/Levantiniella introns, than with the Scleritodermidae (Fig. 5). A HGT from an ancestor of Cinachyrella/Levantiniella species to some Agelasida could account for this result. In all these examples it can therefore be hypothesised that a HGT occurred between distantly related sponge groups. Although the mechanism of intron HGT is unknown at this point, we noted that these donor/receiver species originate from the same regions and share the same habitats (reef, lake or mangrove, see Additional file 3), which is expected to make HGT possible.

Similarities in intron secondary structures of distantly related sponges are further evidence for HGT [43]. Hence, independent insertion events in Tetillidae, Axinellidae and Agelasida were proposed for intron 723 [43]. This is confirmed by secondary structure differences we observed in closely related families (Tetillidae and Scleritodermidae) (Fig. 6, Additional file 2). Additional loops (P9.1e,f), reduced stems (e.g., P9.1d) and the absence of the P6d region in Sceritodermidae (Fig. 6) result in a higher structure similarity to e.g., Axinella polypoides [43], rather than to other Tetillidae structures ([65]; Additional file 2, [82]), which confirms independent insertions of intron 723 in Scleritodermidae and Tetillidae. No major structural differences of intron 723 in the P9 and P6 regions were observed within different species of Cinachyrella (Additional file 2) except for Cinachyrella sp. 4 from
Morocco, which showed reduced P9.1c and P9.1d helices. A few minor differences were also noted between Cinachyrella and L. levantinensis structures (Fig. 2 in [65]): the latter had an additional loop in P5a, a reduced P9.1d and a loop at the end of P6d. Interestingly, the latter two features are also observed in C. sp. 4 (Additional file 2 ), which could be explained by their common origin resulting from a HGT (Fig. 9). The relative similarity of intron 723 between Cinachyrella and Levantiniella is a strong argument in favor of a single insertion event in this clade, which therefore implies at least two losses of intron 723 to account for the two major Cinachyrella/Paratetilla/Amphitethya clades without any intron (Fig. 9). These would be the first reported cases of $\mathrm{mt}$ intron secondary loss in sponges.

For intron 870 no structure differences were observed between Tetilla quirimure (MNRJ 17891) (Fig. 6) and Tetilla radiata (HM032742) [82]. Remarkably, Tetilla japonica (JX177901), which is sister to Tetilla radiata (with a strongly supported node) does not posses intron 870. We therefore assume that Tetilla japonica secondarily lost intron 870 , which would represent another case of mt intron loss in sponges. The structure of intron 870 in Microscleroderma herdmani 3 displays a reduced P6a and an additional P5d region (Fig. 6d) compared to Tetilla radiata / quirimure, which suggests an independent insertion of intron 870 as the most plausible explanation. This is further corroborated by the distant phylogenetic relationship between Tetilla and Scleritodermidae (Fig. 4) and the LAGLIDADG phylogeny (Fig. 7).

Until now VGT was only assumed within sponges [82], but awaited proof with a wider sampling. For the first time our study on 63 Cinachyrella sequences provides conclusive evidence that introns were vertically transmitted due to 1) mostly congruent cox 1 versus intron phylogenies and 2) similarity of secondary structures among closely related species. Introns 714, 723 and 870 have all undergone VGT, but this is especially apparent for intron 723 for which we have the largest sampling (Figs. 5 and 9). VGT for group I introns are also known e.g., from hexacorals (nad5-717 intron, [19]), but is often difficult to ascertain due to the patchy distribution of introns. To conclude, our results demonstrate that introns 714, 723 and 870 undergo VGT, HGT and secondary loss events, and that both VGT and HGT can occur within one genus (e.g., Cinachyrella) (Fig. 9). 


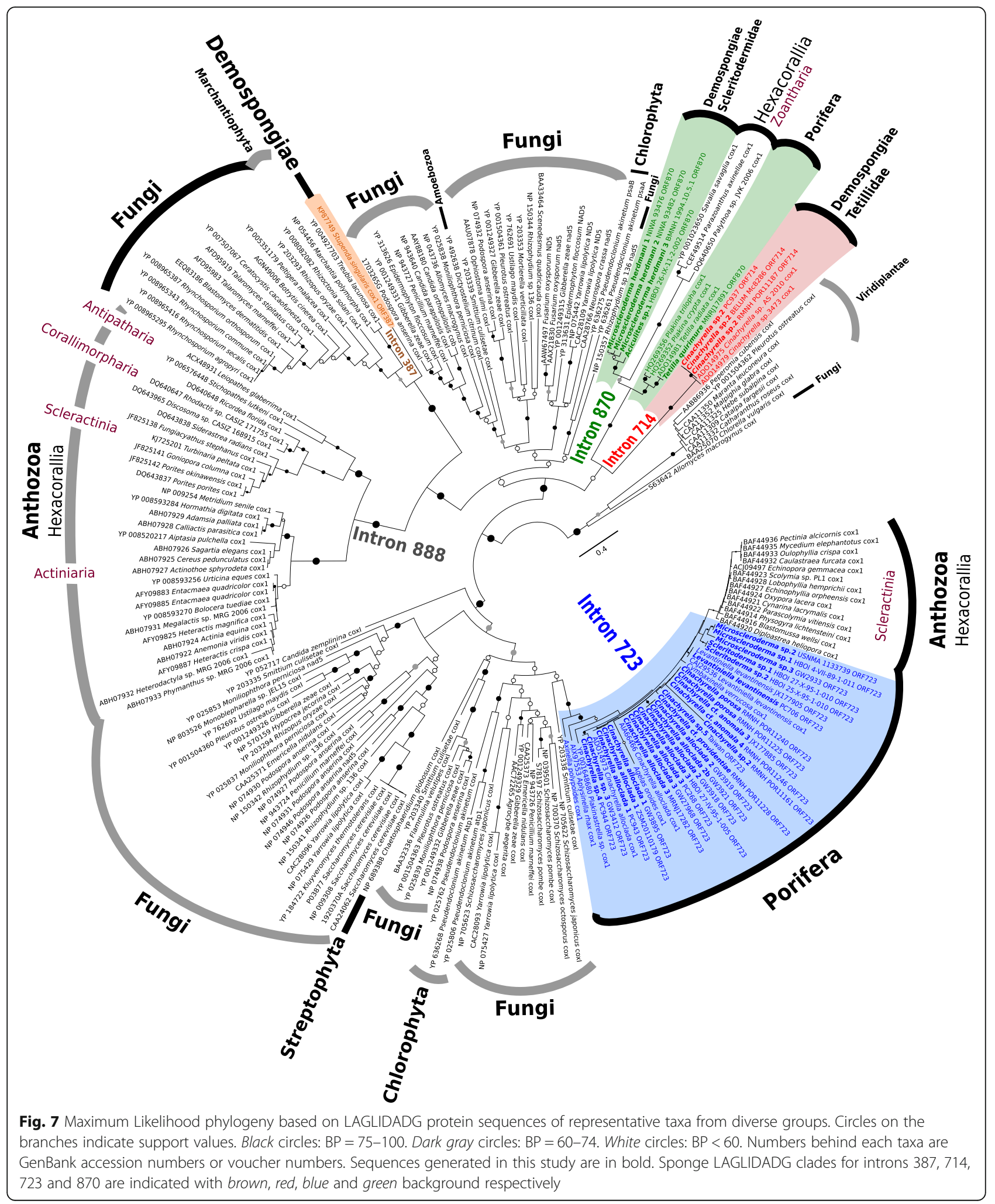




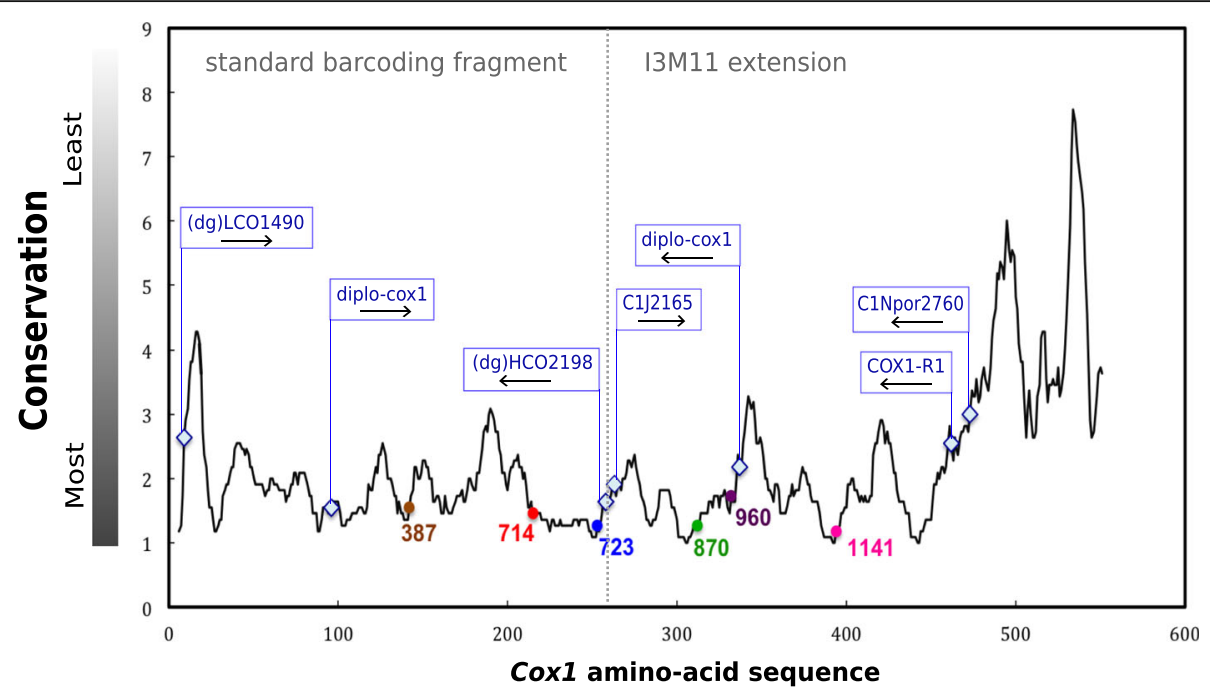

Fig. 8 Conservation profile of the complete translated cox 1 gene for sponges. X-axis indicates the amino acid position along the alignment. Yaxis assigns the number of amino-acid substitutions over a range of 11 aligned positions throughout the alignment. Different intron insertion positions of group I introns (387, 714, 723 and 870) and group II introns (966 and 1141) are plotted on the profile line and color-coded with respect to Fig. 2. Additionally, the 5' position of commonly used sponge barcoding markers is indicated as light blue rhombuses. A detailed list of primers is provided in Additional file 4

\section{Origin of group I introns}

The origins of group I introns has been debated for many eukaryotic organisms (e.g., [63]) including sponges [43]. Fungi are proposed as the primary donor of mt group I introns not only in plants (e.g., [14]), but also in cnidarians [31] and sponges [47, 65, 82]. Placozoa have also been suggested as possible donors in sponges, but only for intron 387 in one species [48]. Sponge-fungal associations, pivotal for such HGT, are well-known for sponges (e.g., [40, 71]). However, only little is known about the specific fungal lineages associated with intron-bearing sponge taxa. For Cinachyrella, however, deep sequencing analysis recently identified Ascomycota as a dominant fungal phylum (Cinachyrella cf. australiensis and Cinachyrella sp. from the China Sea [38]). This is corroborated by data for Cinachyrella alloclada from the Caribbean that showed that the cosmopolitan Phoma sp. (Ascomycota) is the dominant sponge-associated fungus, while nine more ascomycete species were found [5]. Indeed, in the reconstructed LAGLIDADG protein phylogeny (Fig. 7) all intron 723 sequences of Cinachyrella and other sponge taxa display a close relationship to ascomycete intron sequences, but also Viridiplantae (Chlorophyta and Streptophyta). The latter is not surprising, as intron of chlorophytes and Viridiplantae similarly have their origin from Ascomycota [53, 87]. The huge assemblage of group I introns described in fungi increases the chance of a HGT event. When metazoans and plants host one or two group I introns in their cox1, fungus like Ophiocordyceps sinensis contains 21 group I introns within its $\operatorname{cox} 1$ region alone [54].

The non-bilaterian LAGLIDADG-protein sequence dataset (Fig. 7) identifies Scleractinia (stony corals) and Zoantharia (zoanthids) sequences as the only sequences respectively homologous to the sponge introns 723 and 870. Although this is not very well supported, our results show a sister relationship between LAGLIDADG in Scleritodermidae and Zoantharia, which suggests they may have contaminated each other (the direction of the HGT is unclear at this point); alternatively they were contaminated by the same donor, un-sequenced as of today. Because the Scleractinia intron 723 LAGLIDADG sequences are nested within the sponge sequences (Fig. 7), Fukami et al. [27] suggested two alternative scenarios: 1) Scleractinia and sponges have a similar fungi donor which independently transferred intron 723 in each group or 2) HGT events from each other (sponges to coral, or vice versa). However, although intron 723 has a patchy distribution in the Scleractinia [27], the Scleractinia LAGLIDADG sequences form a well supported clade (Fig. 7), which suggests that the origin and therefore the donor must have been the same. Different sponges can be excluded as donors, because otherwise the Scleractinia sequences would be partly mixed with the sponge sequences. Also, the possibility of one single sponge donor is unlikely, because there is no sponge species living in close contact with all these Scleractinia species from the Indo-Pacific and the Atlantic. Thus, we are in favor of a donor, most probably a fungus, which transferred intron 723 to different Scleractinia, while 


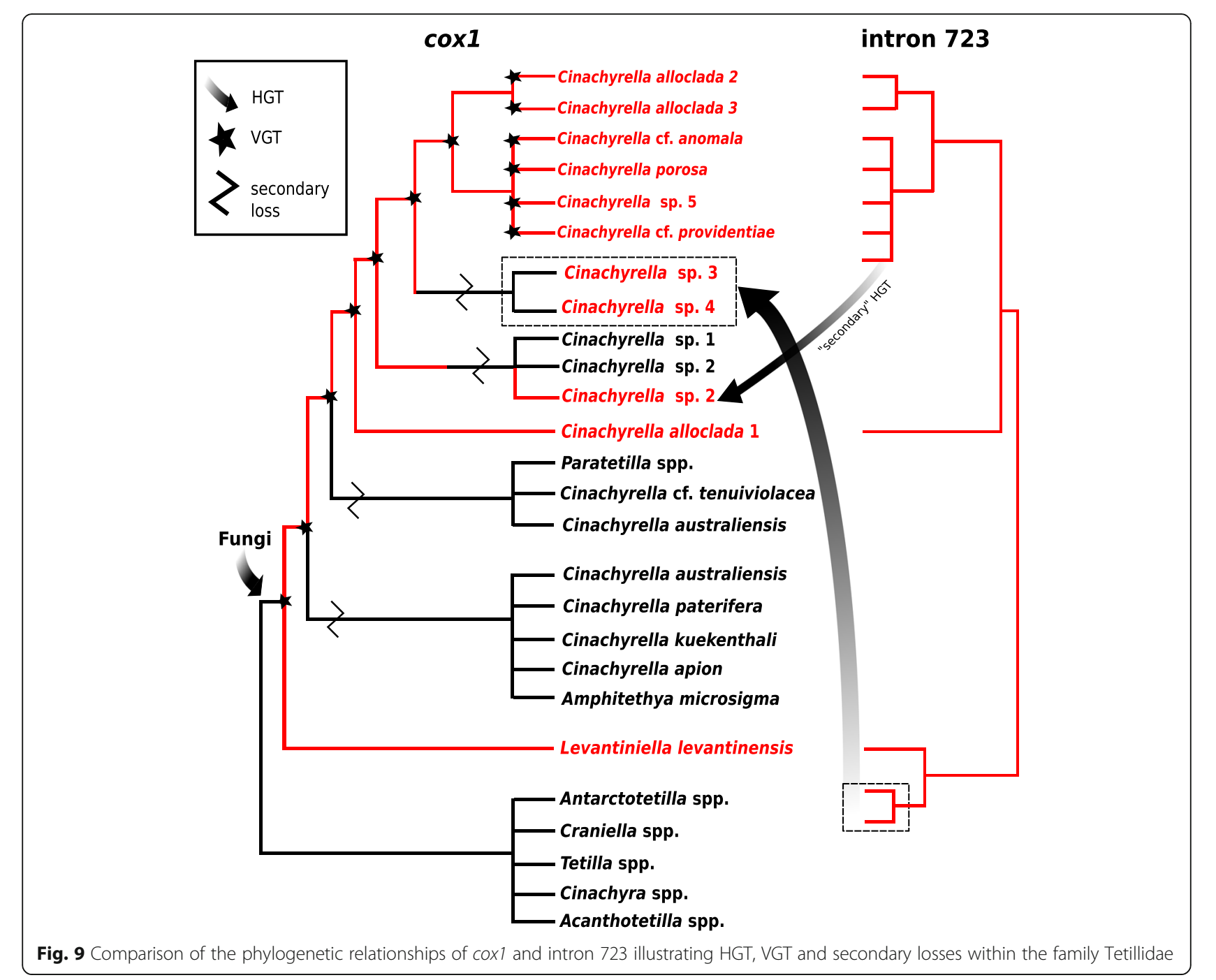

similar donors transferred intron 723 to different sponges. These donors can probably also act as vectors, thereby enabling HGT between Cinachyrella species, as shown above. Intron 387 of Stupenda singularis is the only sponge LAGLIDADG sequence apparently unrelated to any coral LAGLIDADG in the data set, but is closely related to LAGLIDADG found in fungi and Marchantiophyta (liverworts). Liverworts are thought to have received their introns from fungi [58], and the close relationship of the Stupenda singularis LAGLIDADG 387 likewise suggests a fungal origin (see also [47]).

Unfortunately, the origin of sponge intron 714 remains unresolved, since no supported relationship to any of the included taxonomic groups is given. Therefore, we cannot exclude the possibility that group I introns in sponges may originate from an undiscovered and/or un-sequenced sponge-associated symbionts, e.g., fungi, Archaea, Bacteria or dinoflagellates, since all are known to posses group I introns. In particular sponge bacterial symbionts, which can contribute to over $50 \%$ of the sponge biomass [39, 69], may play an essential role as potential intron donors. However, according to our results (Fig. 7) it is unlikely that bacteria, archaea or dinoflagellates are donors, because of the absence of a homologous LAGLIDADG motif. Blastx of intron 714 specified fungi as best hits, therefore fungi remains a good donor candidate for this intron. According to the diversity of habitats of intronbearing sponges, from shallow water (in reefs, marine lakes and mangrove) to the deep sea (Additional file 3), we can hypothesize that the putative intron donor may also be ubiquitous, and present in all these different environments.

\section{Implications for DNA barcoding}

Intron insertion in highly conserved cox 1 regions decreases the possibility of intron elimination, because the removal must be specific in order to avoid any disruption of the protein function. The most widespread intron 
723 is located at the most conserved site in the cox 1 gene (Fig. 8), suggesting this position as an "intron hotspot". In addition to the high number of intron 723 in Tetillidae, we discovered several more intron 714 (Cinachyrella) and intron 870 (Scleritodermidae, Tetilla), both in conserved $\operatorname{cox} 1$ regions. Intron presence in conserved cox1 gene regions has major consequences for other fields of science such as molecular taxonomy. In order to gain a better understanding of locations and conservation of the currently recommended barcoding primers [22, 24, 91], we plotted the $5^{\prime}$ site of each primer on the cox1 conservation profile line (Fig. 8). Interestingly, for the standard barcoding fragment and the I3M11 extension our analysis shows that all previously applied sponge barcoding primers are located in comparatively less conserved regions (Fig. 8). Moreover, our results indicate that group I intron 723 and group II intron 960 are in close proximity to barcoding reverse primer sites (HCO2198 and diplo-cox1) or interrupt the priming regions (intron 723), which corroborates earlier findings from Szitenberg (2010). These findings may partly explain the low ( $25 \%$ mean) amplification success reported for barcoding museum samples using standard barcoding primers [86]. We therefore recommend for future sponge barcoding studies to test the reverse COX1-R1 primer, which is more distant to the intron insertions, instead of the HCO2198 primer (Fig. 8). The COX1-R1 primer, originally designed to amplify the cox 1 of Tetillidae [65], has been shown to successfully amplify cox1 in Poecilosclerida [62], Agelasida and Axinellida [43], Chondrosiida and Dictyoceratida [3], some Astrophorina (P. Cárdenas, unpublished results) and Spirophorina (this study).

\section{Conclusion}

This study provides novel insights into the taxonomic distribution, diversity and mobility of mitochondrial group I introns in sponges, and validates the subclass Spirophorina (Tetractinellida), as an intron hotspot in sponges, notably by increasing the number of Tetillidae introns known by a factor of 5 . We wonder whether this could be linked to a lower mt mutation rate in the Spirophorina with respect to other sponges, as suggested for some intron hotspot fungi groups [44]. We show that co-occurrence of two introns in cox 1 is unique among metazoans, but not uncommon in sponges. However, this combination always associates a potentially active intron with a degenerating one. Earlier hypotheses of HGT were confirmed and for the first time VGT and secondary losses of introns conclusively demonstrated. Consequently, such a high level of HGT in combination with the relative low variation in case of VGT (e.g., intron 723, Fig. 9), rejects any alternative use of $m t$ introns as phylogeographic markers. Since the majority of sponge introns encode a HEG in frame with the 5' exon, activity of those introns is assumed. We further demonstrate that introns are not restricted to shallow water sponge species, but also occur in species from deeper $(\sim 500 \mathrm{~m})$ habitats and extreme environments (mangroves and marine lakes). Conservation profile analysis reveals that all group I and possibly also group II intron insertions in sponges are located within the most conserved regions of their host protein, which may partly explain why they persist in their host genes. At the same time, we show that the currently used sponge barcoding primers are usually located in less conserved regions compared to the introns, but can also overlay intron insertion sites. Therefore, we recommend applying different primers (in particular reverse primers) when standard barcoding primers fail to amplify the $\operatorname{cox} 1$ gene. Finally, our study enhances the support for a fungal origin for the majority of introns in sponges.

\section{Methods \\ Sampling and identification of specimens}

Cinachyrella samples were collected in Florida (U.S.A.) by snorkeling in the seagrass meadows adjacent to the Mote Marine Laboratory/Tropical Research Laboratory (Summerland Key, Florida U.S.A.) and by scuba-diving on the Broward County reef located off Fort Lauderdale $\left(26^{\circ} 10.498,-80^{\circ}\right.$ 05.632). More Cinachyrella spp. were collected in Indonesia by diving on reefs and snorkeling in mangroves and marine lakes in West Papua and East Kalimantan, Indonesia. The remaining material was obtained through collaborators or sampled in several museum collections (Additional file 3). Because of ambiguous sequences or missing data, some Cinachyrella specimens from Szitenberg et al. [81] were successfully re-sequenced (JX177885, JX177886, JX177887 and JX177913). Taxonomic identification to genus and species level was performed by the authors and follows the findings of Carella et al. [11] on Tetillidae. The species Craniella quirimure from Brazil was re-assigned to the genus Tetilla based on the absence of a clear doublelayered cortex. In some cases identification of species was adopted from collections and earlier publications. Numbers were added for lineages of species that could not be recovered as monophyletic and await revision (e.g., C. alloclada 1-3). A detailed list of species origin including collector, voucher numbers and accession numbers, location and depth are provided in Additional file 3. A Cinachyrella cox 1 sequence (including a group I intron) from Taiwan was manually copied from Hsiao [41]. This species was first identified as Cinachyrella australiensis and is identified as Cinachyrella sp. 5 based on our cox 1 CDS phylogeny. One complete cox 1 sequence of Microscleroderma sp. (USNM 1133739) with an intron was kindly provided by D. V. Lavrov 
(Department of Ecology, Evolution, and Organismal Biology, Iowa State University, USA). The higher level demosponge classification follows Morrow \& Cárdenas [56].

\section{Molecular approach}

Genomic DNA was isolated from the choanosome of the sponge tissue by using the NucleoSpin (Machery-Nagel) or the DNeasy (Qiagen) Blood and Tissue Kit according to the manufacturer's protocol. An additional centrifugation step was added before transferring the lysate to the Spin Column in order to avoid any clogging of the membrane, caused by sponge spicules. Quantification of the isolated genomic DNA was performed using a NanoDrop 1000 Spectrophotometer (Thermo Scientific).

Amplification of the partial cox 1 was performed by using different primers and PCR conditions. Detailed information of primers used for each sample is provided in Additional file 4. For most Tetillidae the $\operatorname{cox} 1$ fragment was amplified using the primers LCO1490 [24] and COX1-R1 [65] and for most Scleritodermidae we used the primers diplo-cox1-f1 and diplo-cox1-r1 [52]. For both primer pairs the PCR settings were: $94{ }^{\circ} \mathrm{C}$, 5 min; $\left(94{ }^{\circ} \mathrm{C}, 1 \mathrm{~min} ; 50-52{ }^{\circ} \mathrm{C}, 1: 30 \mathrm{~min} ; 72{ }^{\circ} \mathrm{C}\right.$, 1:30 min $) \times 40$ cycles; $72{ }^{\circ} \mathrm{C}, 10 \mathrm{~min}$. Amplified fragments were visually checked for introns by length on a $1.5 \%$ agarose gel. For the majority of the Cinachyrella samples with introns, we observed an additional nonspecific band at position $\sim 600$ bp of bacteria and fungi cox 1 fragments. Separation of double bands and PCR clean-up was performed using a modified freeze-squeeze method [83] in which $20 \mu \mathrm{l}$ of the PCR product were cut from the gel and stored at $-80{ }^{\circ} \mathrm{C}$ for one hour, followed by a $40 \mathrm{~min}$ centrifugation step at $14,000 \mathrm{rpm}$. The supernatant $(6 \mu \mathrm{l})$ was used for cycle sequencing with different and multiple sequencing primers (Additional file 4) together with BigDye Terminator v3.1 (Applied Biosystems, Forster City, CA, USA) chemicals and sequenced by an ABI 3730 Genetic Analyzer at the Sequencing Service of the Department of Biology (LMU München), or by Macrogen (South Korea).

\section{Positions and secondary structures of group I introns within Tetractinellida}

Insertion sites for each intron were ascertained in an alignment including other intron-bearing sequences $[21,43,82]$. Intron specific positions were defined according to the cox 1 sequence of the sponge Amphimedon queenslandica following Szitenberg et al. [82]. Blast hits and sequence similarity to already published group I intron insertions were used to distinguish between different insertion sites and group I and group II introns. An overview of the different group I (intron 387, 714, 723, 870) insertion sites as well as group II (intron 966 \& 1141) insertion sites is given in Fig. 2. Identification of the HEG for each ORF was conducted by blastp against NCBI Genbank [1]. The class of group I introns (IA, IB, IC, ID or IE) was obtained using the RNAweasel Website http:// megasun.bch.umontreal.ca/RNAweasel/ [49]. Initiation and stop codons of the HEG ORFs were located using the ORF finder as implemented in Geneious v.8.1.8 (www.geneious.com) with the following settings: translation Table 4 (Mold and Protozoan mitochondrial) with start codons ATG, GTG, TTG and ATT [90], minimum size $100 \mathrm{bp}$, including interior ORFs. Although considered as potential start codon in sponge group I introns $[47,65]$, there is actually no evidence that TTA is used in sponges as start codon; it has only been found so far in Trypanosoma [26]. We therefore excluded the TTA start codon in our searches and also revisited the ORFs of previously reported sponge group I introns.

In order to predict the secondary structures of group I introns, we manually converted the given secondary core structures into a dot-bracket notation including pseudoknot informations in square brackets. As secondary structure references, we used Cinachyrella alloclada (HM032738) for intron 723 and Tetilla radiata (HM032742) for intron 870 [82]. In order to ensure the right structure annotation for short variable (mainly P6 and P9) domains, we used Mfold http://unafold.rna.albany.edu/ [93] under the general settings, presupposing the exclusion of additional pseudoknots, which cannot be predicted by this program. Those Mfold structures were then manually converted into a dotbracket notation and implemented to the already established core structure sequence. SeaView v4 [32] was used to align the sequences to their structure annotation. The LAGLIDADG regions were removed from the sequences for further analysis. The rest of the intron sequence together with its structure information, was converted to a ct-format using the Perl-script (2ct.zip) of Voigt et al. [89] (available at http://www.palaeontologie.geo.lmu.de/molpal/ RRNA/index.htm). All secondary structures were visualized in RNAViz 2.0.3 http://rnaviz.sourceforge.net/ [18]. Helix names follow Szitenberg et al. [82].

\section{Tetractinellida phylogenies predicted by cox 1 CDS Sequence alignments and outgroup choice}

Newly generated sequences as well as additional GenBank sequences were manually aligned to the datasets from Szitenberg et al. [81, 82]. Aligned sequences were subsequently controlled for discrepancies and corrected by eye. Two Astrophorina species (Geodia barretti and Alectona millari) were used as outgroups in Tetillidae phylogenetic analyses. Astrophorina has been established as the sister clade of Spirophorina in previous studies $[6,57]$. For the analysis of the tetractinellid phylogeny we chose Halichondria melanodocia and Axinella corrugata, which were already successfully used as outgroups 
in previous studies on the molecular phylogeny of the Tetractinellida (e.g., [47]).

The final cox 1 alignment (excluding intron(s)) of the Tetillidae phylogeny comprised 133 sequences (including the two outgroups), of which 76 were newly generated from this study. The alignment was 1177 bp long, of which 829 bp were constant, 62 bp were parsimony uninformative and $286 \mathrm{bp}$ were parsimony informative. The final cox1 alignment of the Tetractinellida phylogeny constituted 82 sequences (including the two outgroups) of which 33 were newly generated from this study. In total the alignment comprised 1118 bp, of which 642 bp were invariant, 77 bp parsimony uninformative and 399 bp were parsimony informative.

\section{Phylogenetic reconstructions}

Phylogenetic tree reconstructions for both analyses were performed on a parallel version of MrBayes v3.2.4 [64] and RAxML v8.0.26 [78] on a Linux cluster. Bayesian analyses were conducted under the most generalized GTR + G + I evolutionary model, as resulted from jModelTest v.2.1.7 [16]. Analyses were run in two concurrent runs of four Metropolis-coupled Markov-chains (MCMC) for 100,000,000 generations and stopped when the average standard deviation of split frequencies reached below 0.01 . The first 25\% (burn-in) of the sampled trees were removed for further analysis. For both datasets, Maximum Likelihood (ML) and bootstrap analyses (1,000 replicates) under the GTR + G model as resulted from jModelTest v.2.1.7 [16] were performed. Tree topologies from Bayesian and ML analyses were compared and visualized using Figtree v1.4.2 http://tree.bio.ed.ac.uk/software/figtree/.

\section{Phylogenetic inference based on intron + LAGLIDADG sequences}

In order to test for vertical transmission of group I introns (including both LAGLIDADG and the non-coding regions) in the genus Cinachyrella, we conducted phylogenetic analyses on separate datasets respectively including all sponge introns 723,714 and 870 . For the analysis of intron 723, we included 74 sequences of which 63 belong to the genus Cinachyrella. One taxon Aciculites sp. 1 (HBOI 26-IX-11-2-002), was excluded from this analysis, as no putative HEG were detected in the intron. The final intron 723 alignment was 1167 bp long, of which 488 bp were constant, 307 bp parsimony uninformative and 372 bp parsimony informative. The final intron 714 dataset included 13 sequences and was $946 \mathrm{bp}$ long, of which 891 bp were constant, $49 \mathrm{bp}$ were parsimony uninformative and $6 \mathrm{bp}$ were parsimony informative. As an outgroup for both analysis we used the introns of Plakinastrella sp. (NC 010217), a species that belongs to a different sponge class (Fig. 1). The final alignment of intron 870 contained 12 taxa and was 974 bp long, of which 615 pb were constant, 46 bp were parsimony uninformative and 313 bp parsimony informative. Plakina trilopha (HQ269356) and P. crypta (HQ269352) which belong to a different sponge class (Fig. 1) were used as outgroups. Phylogenetic tree reconstructions were performed as described above for the cox 1 exon phylogeny.

In order to test whether the incongruencies between the exon and the intron/HE phylogeny were significant, we performed a series of Shimodaira-Hasegawa (SH) tests [75] as implemented in RAxML [78] on the exon tree against ML topologies constrained towards the intron tree topology. Constraints were inferred with Mesquite v.3.10 [55].

\section{Phylogenetic reconstructions based on LAGLIDADG protein sequences of group I introns}

In order to investigate the evolutionary origins of the putative LAGLIDADG encoding introns (387, 714, 723 and 870 ) in sponges the newly generated sequences were added to the LAGLIDADG dataset by Huchon et al. [43]. Additionally, we included 12 fungal and two Marchantiophyta LAGLIDADG sequences resulting from Blastp hits of the sponge LAGLIDADG for intron 387 (Table 2, [47]). Subsequently, MAFFT v.7 [46] under the L-INS-I algorithm was used to generate the protein alignment. The resulting alignment contains sequences of fungi, plants, cnidarians and sponges. Here, 291 amino-acids (aa) out of 1278 aa were parsimonyuninformative variable characters, 729 aa were constant and 708 parsimony-informative. As a result, we manually corrected the LAGLIDADG alignment. Parts with more than approximately $50 \%$ of missing data were removed manually using the custom site set selection tool in SeaView. The final alignment was 317 amino-acids long, of which one character was constant and two variable characters were parsimony-uninformative. The rest of the 314 characters were phylogenetically informative. The maximum likelihood (ML) analysis was performed using RAxML v8.0.26 [78] on a Linux cluster with 1,000 bootstrap repeats. Using ProtTest 3.4 [15] the best evolutionary model was found to be $\mathrm{VT}+\mathrm{I}+\mathrm{Gamma}+\mathrm{F}$. However, for the RAxML analysis we excluded the invariant parameter (I) from the model, as it is not recommended to use both gamma (G) and invariant (I) parameters among site-rate variations according to the RAxML manual. No root for the tree was specified, as it was not needed for our purpose.

\section{Compilation of the conservation profile}

A conservation profile was calculated from a cox 1 protein alignment dataset compiled from the demosponge sequences from [90], complemented by Homoscleromorpha 
sequences from [29]. The final protein alignment consisted of 58 sequences and 556 characters. The conservation profile was made following Swithers et al. [80] using the same perl script (made available in the supplementary material of Swithers et al. [80]) but with a slightly modification to allow ' $\mathrm{X}$ ' characters in the alignment and calculation. The $5^{\prime}$ position of the common barcoding markers as well as all sponge intron insertion positions were plotted on the profile line.

\section{Additional files}

Additional file 1: Intron 714 and 870 phylogenies. (PDF $38 \mathrm{~kb}$ )
Additional file 2: Predicted secondary structure of introns from different
Cinachyrella species. (PDF $86 \mathrm{~kb}$ )
Additional file 3: Metadata of samples used in this study. (XLSX $85 \mathrm{~kb}$ )
Additional file 4: PCR and sequencing primers used in this study.
$(\mathrm{XLS} 80 \mathrm{~kb})$

\section{Acknowledgements}

We greatly acknowledge Dennis V. Lavrov (lowa State University, USA) for sharing the Microscleroderma sp. 2 cox 1 intron sequence and Oliver Voigt (Dept. of Earth- \& Environmental Sciences, LMU Munich, Germany) for providing scripts and support for secondary structure analysis. We greatly thank Eduardo Hajdu and Cristina Castello-Branco (Universidade Federal Do Rio De Janeiro, Brasil), Carsten Lüter (Museum für Naturkunde Berlin, Germany), Helmut Lehnert (Zoologische Staatssammlung München, Germany), Sigal Shefer and Yaniv Aluma (Tel Aviv University), Sadie Mills and Kareen Schnabel (National Institute of Water and Atmospheric Research, Wellington, New Zealand), Sara Griffiths (University of Manchester, UK), Cécile Debitus (IRD, Institut de Recherche pour le Développement, Marseille, France)France), Christopher J. Freeman (Smithsonian Marine Station, Fort Pierce, USA), Belkassem El Amraoui (University Ibn Zohr, Taroudant, Morocco), Nadia Santodomingo (Natural History Museum, London), Nicole J. De Voogd (Naturalis Biodiversity Center), Yosephine Tuti (Indonesian National Institute of Sciences) and the governments of Ecuador, Commonwealth of the Bahamas, Panama, and Martinique (France) for sampling, sharing material and help in the collections. We thank Kyle Roebuck, Nidhi Vijayan and Marissa Wickes with help in sample preparation and shipping from Florida. We thank the Systematic Biology lab (Dept. of Organismal Biology, Uppsala University, Sweden) and Gabrielle Büttner and Simone Schätzle (Dept. of Earth- \& Environmental Sciences, LMU Munich, Germany) for sequencing assistance. Finally, we want to thank the three anonymous reviewers for their helpful comments and suggestions.

\section{Funding}

This work was funded by the German Science Foundation (DFG) (grant number DFG ER 611/3-1, DFG Wo896/15-1); LMUMentoring Program; HELGE AX:Son JOHNSON STIFTELSE (Sweden), Inez Johanssons HT2012 (Uppsala University, Sweden) and Netherlands Organisation for Scientific Research (NWO) Veni\#863.14.020.

\section{Availability of data and material}

New sequences from this study are stored at the European Nucleotide Archive (ENA) under the accession numbers LT628277-LT628366. All alignments generated during the current study are freely available at OpenDataLMU (doi:10.5282/ubm/data.98).

\section{Authors' contributions}

AS and PC conceived and designed the study. AS carried out PCR, sequencing, phylogenetic analyses and predicted the secondary structures. AS, LEB, MK, PC, and SAP identified the specimens. All authors contributed with samples and reagents. AS drafted the manuscript and figures. PC, JVL, SAP, GW and DE assisted in revising the MS. All authors approved the final version of the manuscript.

\section{Competing interests}

The authors declare that they have no competing interests.

\section{Consent for publication \\ Not applicable.}

\section{Ethics approval and consent to participate}

This study did not include protected or endangered species and requires no ethical approval. Collection permits in Indonesia were provided by the Indonesian Institute of Sciences (LIPI) and the Indonesian State Ministry of Research and Technology (RISTEK) to LEB: 0094/frp/sm/v/2009, 1810/FRP/SM/ VIII/2008, 098/SIP/FRP/SMN/2011). Collecting permits in Florida were provided by the Florida Fish and Wildlife Conservation Commission by a valid fishing license.

\section{Publisher's Note}

Springer Nature remains neutral with regard to jurisdictional claims in published maps and institutional affiliations.

\section{Author details}

${ }^{1}$ Department of Earth- \& Environmental Sciences, Palaeontology and Geobiology, Ludwig-Maximilians-Universität München, Richard-Wagner-Str. 10, 80333 Munich, Germany. ${ }^{2}$ Halmos College of Natural Sciences and Oceanography, Nova Southeastern University, Dania Beach, FL 33004, USA. ${ }^{3}$ Marine Animal Ecology, Wageningen University \& Research Centre, P.O. Box 3700AH, Wageningen, The Netherlands. ${ }^{4}$ Naturalis Biodiversity Center, Marine Zoology Department, PO Box 95172300RA, Leiden, The Netherlands. ${ }^{5}$ National Centre for Aquatic Biodiversity and Biosecurity, National Institute of Water and Atmospheric Research, P.O. Box 109-695Newmarket, Auckland, New Zealand. ${ }^{6}$ Harbor Branch Oceanographic Institute-Florida Atlantic University, 5600 U.S. 1 North, Ft Pierce, FL 34946, USA. ${ }^{7}$ SNSB - Bavarian State Collections of Palaeontology and Geology, Richard-Wagner Str. 10, 80333 Munich, Germany. ${ }^{8}$ GeoBio-CenterLMU, Ludwig-Maximilians-Universität München, Richard-Wagner Str. 10, 80333 Munich, Germany. ${ }^{9}$ Department of Medicinal Chemistry, Division of Pharmacognosy, BioMedical Center, Uppsala University, Husargatan 3, 75123 Uppsala, Sweden.

Received: 15 September 2016 Accepted: 28 February 2017

Published online: 20 March 2017

\section{References}

1. Altschul SF, Madden TL, Schaffer AA, Zhang JH, Zhang Z, Miller W, Lipman DJ. Gapped BLAST and PSI-BLAST: a new generation of protein database search programs. Nucleic Acids Res. 1997;25:3389-402.

2. Belfort M, Perlman PS. Mechanisms of intron mobility. J Biol Chem. 1995; 270:30237-40

3. Belinky F, Szitenberg A, Goldfarb I, Feldstein T, Wörheide G, Ilan M, Huchon D. ALG11 - A new variable DNA marker for sponge phylogeny: Comparison of phylogenetic performances with the $18 \mathrm{~S}$ rDNA and the COI gene. Mol Phylogenet Evol. 2012;63:702-13.

4. Bilewitch JP, Degnan SM. A unique horizontal gene transfer event has provided the octocoral mitochondrial genome with an active mismatch repair gene that has potential for an unusual self-contained function. BMC Evol Biol. 2011;11:228.

5. Bolaños J, Fernando De León L, Ochoa E, Darias J, Raja HA, Shearer CA, Miller AN, Vanderheyden P, Porras-Alfaro A, Caballero-George C. Phylogenetic diversity of sponge-associated fungi from the Caribbean and the Pacific of Panama and their in vitro effect on angiotensin and endothelin receptors. Mar Biotechnol. 2015;17:533-46.

6. Borchiellini C, Chombard C, Manuel M, Alivon E, Vacelet J, Boury-Esnault N. Molecular phylogeny of Demospongiae: implications for classification and scenarios of character evolution. Mol Phylogenet Evol. 2004;32:823-37.

7. Burger G, Forget L, Zhu Y, Gray MW, Lang BF. Unique mitochondrial genome architecture in unicellular relatives of animals. Proc Natl Acad Sci U S A. 2003;100:892-7.

8. Burger G, Saint-Louis D, Gray MW, Lang BF. Complete sequence of the mitochondrial DNA of the red alga Porphyra purpurea. Cyanobacterial introns and shared ancestry of red and green algae. Plant Cell. 1999;11: 1675-94.

9. Burger $G$, Yan $Y$, Javadi $P$, Lang BF. Group I-intron trans-splicing and mRNA editing in the mitochondria of placozoan animals. Trends Genet. 2009;25:381-6. 
10. Cárdenas P, Menegola C, Rapp HT, Cristina DM. Morphological description and DNA barcodes of shallow-water Tetractinellida (Porifera: Demospongiae) from Bocas del Toro, Panama, with description of a new species. Zootaxa. 2009;2276:1-39.

11. Carella M, Agell G, Cárdenas P, Uriz MJ. Phylogenetic reassessment of Antarctic Tetillidae (Demospongiae, Tetractinellida) reveals new genera and genetic similarity among morphologically distinct species. PLoS One. 2016; 11(8):e0160718.

12. Chan H-M. Self-splicing of Group I Intron of the Mitochondrial Genome of the Sponge, Cinachyrella australiensis. Kahosiung: Master Thesis: National Sun Yat-sen University; 2009. http://etd.lib.nsysu.edu.tw/ETD-db/ETD-search/ view_etd?URN=etd-0819109-130742 Accessed 20 Mar 2016.

13. Chen I-P, Tang C-Y, Chiou C-Y, Hsu J-H, Wei NV, Wallace CC, Muir P, Wu H, Chen CA. Comparative analyses of coding and noncoding DNA regions indicate that Acropora (Anthozoa: Scleractina) possesses a similar evolutionary tempo of nuclear vs. mitochondrial genomes as in plants. Mar Biotechnol. 2009;11:141-52

14. Cho Y, Qiu YL, Kuhlman P, Palmer JD. Explosive invasion of plant mitochondria by a group I intron. Proc Natl Acad Sci U S A. 1998;95:14244-9.

15. Darriba D, Taboada GL, Doallo R, Posada D. ProtTest 3: fast selection of bestfit models of protein evolution. Bioinformatics. 2011;27:1164-5.

16. Darriba D, Taboada GL, Doallo R, Posada D. jModelTest 2: more models, new heuristics and parallel computing. Nat Methods. 2012;9:772.

17. Dellaporta SL, Xu A, Sagasser S, Jakob W, Moreno MA, Buss LW, Schierwater B. Mitochondrial genome of Trichoplax adhaerens supports Placozoa as the basal lower metazoan phylum. Proc Natl Acad Sci U S A. 2006;103:8751-6.

18. De Rijk P, Wuyts J, De Wachter R. RnaViz 2: an improved representation of RNA secondary structure. Bioinformatics. 2003;19:299-300.

19. Emblem Å, Karlsen BO, Evertsen J, Johansen SD. Mitogenome rearrangement in the cold-water scleractinian coral Lophelia pertusa (Cnidaria, Anthozoa) involves a long-term evolving group I intron. Mol Phylogenet Evol. 2011;61:495-503.

20. Emblem $\AA$, Okkenhaug S, Weiss ES, Denver DR, Karlsen BO, Moum T, Johansen SD. Sea anemones possess dynamic mitogenome structures. Mol Phylogenet Evol. 2014;75:184-93.

21. Erpenbeck D, Aryasari R, Hooper JNA, Wörheide G. A Mitochondrial Intron in a Verongid Sponge. J Mol Evol. 2014;80:13-7.

22. Erpenbeck D, Hooper JNA, Wörheide G. CO1 phylogenies in diploblasts and the "Barcoding of Life"- are we sequencing a suboptimal partition? Mol Ecol Notes. 2006:6:550-3

23. Férandon C, Moukha S, Callac P, Benedetto J-P, Castroviejo M, Barroso G. The Agaricus bisporus cox1 Gene: The Longest Mitochondrial Gene and the Largest Reservoir of Mitochondrial Group I Introns. PLoS One. 2010;5:e14048.

24. Folmer $\mathrm{O}$, Black M, Hoeh W, Lutz R, Vrijenhoek R. DNA primers for amplification of mitochondrial cytochrome c oxidase subunit I from diverse metazoan invertebrates. Mol Mar Biol Biotechnol. 1994;3:294-9.

25. Fontaine J-M, Rousvoal S, Leblanc C, Kloareg B, Loiseau-de GS. The Mitochondrial LSU rDNA of the Brown Alga Pylaiella littoralis Reveals a- Proteobacterial Features and is Split by Four Group IIB Introns with an Atypical Phylogeny. J Mol Biol. 1995;251:378-89.

26. Fox TD. Natural variation in the genetic code. Annu Rev Genet. 1987;21:67-91.

27. Fukami H, Chen CA, Chiou CY, Knowlton N. Novel group I introns encoding a putative homing endonuclease in the mitochondrial cox1 gene of Scleractinian corals. J Mol Evol. 2007;64:591-600.

28. Galtier N. The intriguing evolutionary dynamics of plant mitochondrial DNA. BMC Biol. 2011;9:1-3.

29. Gazave E, Lapébie P, Renard E, Vacelet J, Rocher C, Ereskovsky AV, Lavrov DV, Borchiellini C. Molecular Phylogeny Restores the Supra-Generic Subdivision of Homoscleromorph Sponges (Porifera, Homoscleromorpha). PLoS One. 2010:5:e14290.

30. Goddard MR, Burt A. Recurrent invasion and extinction of a selfish gene. Proc Natl Acad Sci U S A. 1999:96:13880-5.

31. Goddard MR, Leigh J, Roger AJ, Pemberton AJ. Invasion and persistence of a selfish gene in the Cnidaria. PLoS One. 2006;1:e3.

32. Gouy M, Guindon S, Gascuel O. SeaView version 4: A multiplatform graphical user interface for sequence alignment and phylogenetic tree building. Mol Biol Evol. 2010;27:221-4.

33. Hafez M, Hausner G, Bonen L. Homing endonucleases: DNA scissors on a mission. Genome. 2012;55:553-69.

34. Haugen $P$, Bhattacharya D. The spread of LAGLIDADG homing endonuclease genes in rDNA. Nucleic Acids Res. 2004;32:2049-57.
35. Haugen P, Simon DM, Bhattacharya D. The natural history of group I introns Trends Genet. 2005;21:111-9.

36. Hausner G. Introns, Mobile Elements, and Plasmids. In: Organelle Genetics. Heidelberg: Springer Berlin Heidelberg; 2012. p. 329-57.

37. Hellberg ME. No variation and low synonymous substitution rates in coral mtDNA despite high nuclear variation. BMC Evol Biol. 2006;6:24

38. He L, Liu F, Karuppiah V, Ren Y, Li Z. Comparisons of the fungal and protistan communities among different marine sponge holobionts by pyrosequencing. Microb Ecol. 2014;67:951-61.

39. Hentschel U, Piel J, Degnan SM, Taylor MW. Genomic insights into the marine sponge microbiome. Nat Rev Microbiol. 2012;10:641-54.

40. Höller U, Wright AD, Matthee GF, Konig GM, Draeger S, Aust H-J, Schulz B. Fungi from marine sponges: diversity, biological activity and secondary metabolites. Mycol Res. 2000;104:1354-65.

41. Hsiao S-T. Genomic Analyses of the Complete Mitochondrial DNA Sequence of the Sponge, Cinachyrella australiensis (Demospongiae: Spirophorida). Kahosiung: Master Thesis: National Sun Yat-sen University; 2005. http://etd. lib.nsysu.edu.tw/ETD-db/ETD-search/view_etd?URN=etd-0707105-161158. Accessed 20 Mar 2016.

42. Huang D, Meier R, Todd PA, Chou LM. Slow mitochondrial COI sequence evolution at the base of the metazoan tree and its implications for DNA barcoding. J Mol Evol. 2008;66:167-74.

43. Huchon D, Szitenberg A, Shefer S, Ilan M, Feldstein T. Mitochondrial group I and group II introns in the sponge orders Agelasida and Axinellida. BMC Evol Biol. 2015;15:278.

44. Jalalzadeh B, Saré IC, Férandon C, Callac P, Farsi M, Savoie J-M, Barroso G. The intraspecific variability of mitochondrial genes of Agaricus bisporus reveals an extensive group I intron mobility combined with low nucleotide substitution rates. Curr Genet. 2015;61:87-102.

45. Johansen S, Johansen T, Haugli F. Structure and evolution of myxomycete nuclear group I introns: a model for horizontal transfer by intron homing. Curr Genet. 1992:22:297-304

46. Katoh K, Standley DM. MAFFT Multiple sequence alignment software version 7: Improvements in performance and usability. Mol Biol Evol. 2013;30:772-80.

47. Kelly M, Cárdenas P. An unprecedented new genus and family of Tetractinellida (Porifera, Demospongiae) from New Zealand's Colville Ridge, with a new type of mitochondrial group I intron. Zool J Linn Soc. 2016;177:335-52.

48. Kohn AB, Citarella MR, Kocot KM, Bobkova Y, Halanych KM, Moroz LL. Rapid evolution of the compact and unusual mitochondrial genome in the ctenophore, Pleurobrachia bachei. Mol Phylogenet Evol. 2012;63:203-7.

49. Lang BF, Laforest MJ, Burger G. Mitochondrial introns: a critical view. Trends Genet. 2007;23:119-25.

50. Lavrov DV. Mitochondrial Genomes of Two Demosponges Provide Insights into An Early Stage of Animal Evolution. Mol Biol Evol. 2005;22:1231-9.

51. Lavrov DV, Adamski M, Chevaldonné P, Adamska M. Extensive Mitochondrial mRNA Editing and Unusual Mitochondrial Genome Organization in Calcaronean Sponges. Curr Biol. 2016;26:86-92.

52. Lavrov D, Wang X, Kelly M. Reconstructing ordinal relationships in the Demospongiae using mitochondrial genomic data. Mol Phylogenet Evol. 2008;49:111-24

53. Lindstrom SC, Pistolic J. Detection of a group I (IE) fungal intron in the green algal genus Urospora (Ulvophyceae). J Phycol. 2005;41:359-65.

54. Li Y, Hu XD, Yang R-H, Hsiang T, Wang K, Liang D-Q, Liang F, Cao D-M, Zhou F, Wen G, et al. Complete mitochondrial genome of the medicinal fungus Ophiocordyceps sinensis. Sci Rep. 2015;5:13892.

55. Maddison WP, Maddison DR. Mesquite: a modular system for evolutionary analyses. 2016. Version 3.10 http://mesquiteproject.org.

56. Morrow C, Cárdenas P. Proposal for a revised classification of the Demospongiae (Porifera). Front. Zool. 2015;12:1-27.

57. Nichols S. An evaluation of support for order-level monophyly and interrelationships within the class Demospongiae using partial data from the large subunit rDNA and cytochrome oxidase subunit I. Mol Phylogenet Evol. 2005;34:81-96.

58. Ohta E, Oda K, Yamato K, Nakamura Y, Takemura M, Nozato N, Akashi K, Ohyama K, Michel F. Group I introns in the liverwort mitochondrial genome: the gene coding for subunit 1 of cytochrome oxidase shares five intron positions with its fungal counterparts. Nucleic Acids Res. 1993;21:1297-305

59. Osigus HJ, Eitel M, Bernt M, Donath A, Schierwater B. Mitogenomics at the base of Metazoa. Mol Phylogenet Evol. 2013;69:339-51. 
60. Palmer JD, Adams KL, Cho Y, Parkinson CL, Qiu YL, Song K. Dynamic evolution of plant mitochondrial genomes: mobile genes and introns and highly variable mutation rates. Proc Natl Acad Sci U S A. 2000;97:6960-6.

61. Redmond NE, Morrow CC, Thacker RW, Diaz MC, Boury-Esnault N, Cárdenas P, Hajdu E, Lobo-Hajdu G, Picton BE, Pomponi SA, et al. Phylogeny and Systematics of Demospongiae in Light of New Small-Subunit Ribosomal DNA (18S) Sequences. Integr Comp Biol. 2013;53:388-415.

62. Reveillaud J, van Soest R, Derycke S, Picton B, Rigaux A, Vanreusel A. Phylogenetic relationships among NE Atlantic Plocamionida Topsent (1927) (Porifera, Poecilosclerida): Under-estimated diversity in reef ecosystems. PLoS One. 2011;6:e16533.

63. Rodríguez-Trelles F, Tarrío R, Ayala FJ. Origins and evolution of spliceosomal introns. Annu Rev Genet. 2006;40:47-76.

64. Ronquist F, Teslenko M, van der Mark P, Ayres DL, Darling A, Höhna S, Larget B, Liu L, Suchard MA, Huelsenbeck JP. MrBayes 3.2: Efficient Bayesian Phylogenetic Inference and Model Choice across a Large Model Space. Syst Biol. 2012;61:539-42.

65. Rot C, Goldfarb I, Ilan M, Huchon D. Putative cross-kingdom horizontal gene transfer in sponge (Porifera) mitochondria. BMC Evol Biol. 2006;6:71.

66. Sanchez-Puerta MV, Abbona CC, Zhuo S, Tepe EJ, Bohs L, Olmstead RG, Palmer JD. Multiple recent horizontal transfers of the cox 1 intron in Solanaceae and extended co-conversion of flanking exons. BMC Evol Biol. 2011;11:277

67. Sanchez-Puerta MV, Cho Y, Mower JP, Alverson AJ, Palmer JD. Frequent, phylogenetically local horizontal transfer of the cox1 group I Intron in flowering plant mitochondria. Mol Biol Evol. 2008;25:1762-77.

68. Santamaria M, Vicario S, Pappadà G, Scioscia G, Scazzocchio C, Saccone C. Towards barcode markers in Fungi: an intron map of Ascomycota mitochondria. BMC Bioinformatics. 2009;10:1-13.

69. Santavy DL, Willenz P, Colwell RR. Phenotypic study of bacteria associated with the caribbean sclerosponge, Ceratoporella nicholsoni. Appl Environ Microbiol. 1990;56:1750-62.

70. Schäfer B. Genetic conservation versus variability in mitochondria: the architecture of the mitochondrial genome in the petite-negative yeast Schizosaccharomyces pombe. Curr Genet. 2003:43:311-26.

71. Schmitt S, Tsai P, Bell J, Fromont J, llan M, Lindquist N, Perez T, Rodrigo A, Schupp PJ, Vacelet J, et al. Assessing the complex sponge microbiota: core, variable and species-specific bacterial communities in marine sponges. ISME J. 2012;6:564-76.

72. Schuster A, Erpenbeck D, Pisera A, Hooper J, Bryce M, Fromont J, Wörheide G. Deceptive Desmas: Molecular Phylogenetics Suggests a New Classification and Uncovers Convergent Evolution of Lithistid Demosponges. PLoS One. 2015;10:e116038.

73. Sellem $\mathrm{CH}$, Belcour L. Intron open reading frames as mobile elements and evolution of a group I intron. Mol Biol Evol. 1997;14:518-26.

74. Shearer TL, Van Oppen MJH, Romano SL, Wörheide G. Slow mitochondrial DNA sequence evolution in the Anthozoa (Cnidaria). Mol Ecol. 2002;11:2475-87.

75. Shimodaira H, Hasegawa M. Multiple comparisons of log-likelihoods with applications to phylogenetic inference. Mol Biol Evol. 1999;16:1114-6.

76. Signorovitch AY, Buss LW, Dellaporta SL. Comparative genomics of large mitochondria in placozoans. PLoS Genet. 2007;3:e13.

77. Smith DR, Kayal E, Yanagihara AA, Collins AG, Pirro S, Keeling PJ. First complete mitochondrial genome sequence from a box jellyfish reveals a highly fragmented linear architecture and insights into telomere evolution. Genome Biol Evol. 2012;4:52-8.

78. Stamatakis A. RAxML version 8: a tool for phylogenetic analysis and postanalysis of large phylogenies. Bioinformatics. 2014;30:1312-3.

79. Stampar SN, Maronna MM, Kitahara MV, Reimer JD, Morandini AC. FastEvolving Mitochondrial DNA in Ceriantharia: A Reflection of Hexacorallia Paraphyly? PLoS One. 2014;9:e86612.

80. Swithers KS, Senejani AG, Fournier GP, Gogarten JP. Conservation of intron and intein insertion sites: implications for life histories of parasitic genetic elements. BMC Evol Biol. 2009;9:303.

81. Szitenberg A, Becking LE, Vargas S, Fernandez JCC, Santodomingo N, Wörheide G, Ilan M, Kelly M, Huchon D. Phylogeny of Tetillidae (Porifera, Demospongiae, Spirophorida) based on three molecular markers. Mol Phylogenet Evol. 2013;67:509-19.

82. Szitenberg A, Rot C, Ilan M, Huchon D. Diversity of sponge mitochondria introns revealed by cox 1 sequences of Tetillidae. BMC Evol Biol. 2010;10:288.
83. Tautz D, Renz M. An optimized freeze-squeeze method for the recovery of DNA fragments from agarose gels. Anal Biochem. 1983;132:14-9.

84. Vallès $Y$, Halanych KM, Boore JL. Group II introns break new boundaries: presence in a bilaterian's genome. PLoS One. 2008;3:e1488.

85. Van Oppen MJ, Olsen JL, Stam WT. Evidence for independent acquisition of group I introns in green algae. Mol Biol Evol. 1993;10:1317-26.

86. Vargas S, Schuster A, Sacher K, Büttner G, Schätzle S, Läuchli B, Hall K, Hooper JNA, Erpenbeck D, Wörheide G. Barcoding Sponges: An Overview Based on Comprehensive Sampling. PLoS One. 2012;7:e39345.

87. Vaughn JC, Mason MT, Sper-Whitis GL, Kuhlman P, Palmer JD. Fungal origin by horizontal transfer of a plant mitochondrial group I intron in the chimeric cox1 gene of Peperomia. J Mol Evol. 1995;41:563-72.

88. Vicens Q, Cech TR. Atomic level architecture of group I introns revealed. Trends Biochem Sci. 2006;31:41-51.

89. Voigt O, Erpenbeck D, Wörheide G. Molecular evolution of rDNA in early diverging Metazoa: First comparative analysis and phylogenetic application of complete SSU rRNA secondary structures in Porifera. BMC Evol Biol. 2008;8:69.

90. Wang $X$, Lavrov D. Seventeen new complete mtDNA sequences reveal extensive mitochondrial genome evolution within the Demospongiae. PLoS One. 2008:3:e2723.

91. Wörheide G, Erpenbeck D, Menke C. The Sponge Barcoding Project: aiding in the identification and description of poriferan taxa. In: Custódio MR, Lôbo-Hajdu G, Hajdu E, Muricy G, editors. Porifera research: biodiversity, innovation and sustainability, vol. 28. Série Livros: Rio de Janeiro; 2007. p. 123-8.

92. Yamada T, Tamura K, Aimi T, Songsri P. Self-splicing group I introns in eukaryotic viruses. Nucleic Acids Res. 1994;22:2532-7.

93. Zuker M. Mfold web server for nucleic acid folding and hybridization prediction. Nucleic Acids Res. 2003;31:3406-15.

\section{Submit your next manuscript to BioMed Central and we will help you at every step:}

- We accept pre-submission inquiries

- Our selector tool helps you to find the most relevant journal

- We provide round the clock customer support

- Convenient online submission

- Thorough peer review

- Inclusion in PubMed and all major indexing services

- Maximum visibility for your research

Submit your manuscript at www.biomedcentral.com/submit
C Biomed Central 